\title{
The self-assembly of $\mathrm{Ag}^{+} /\left[\mathrm{PW}_{11} \mathrm{NbO}_{40}\right]^{4-}$ complexes in non- aqueous solutions
}

Alexandra A. Shmakovat, Alexey S. Berezint, Pavel A. Abramov*t,ll, Maxim N.

\section{Sokolovt,}

† Nikolayev Institute of Inorganic Chemistry, 3 Akad. Lavrentiev Ave. 630090, Russia;

II South Ural State University, Prospekt Lenina, 76, 454080, Chelyabinsk, Russia;

‡ Novosibirsk State University, 2 Pirogova str., 630090, Novosibirsk, Russia;

\section{Supporting information}

\section{Table of content}

Experimental Section .3

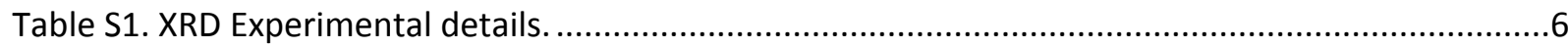

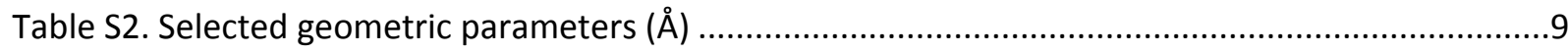

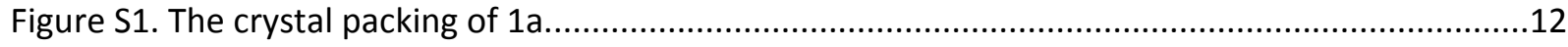

Figure S2. Comparison of observed and calculated PXRD patterns for 1 ....................................13

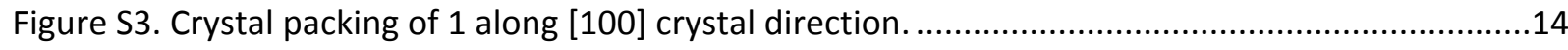

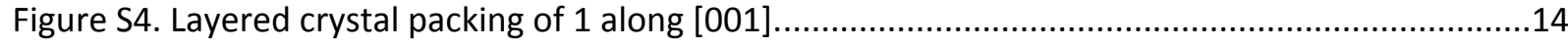

Figure S5. The UV-VIS spectrum of 1a in DMF. The absorption band at $267 \mathrm{~nm}$ overlaps with absorption of DMF which gives incorrect profile of the band..................................................

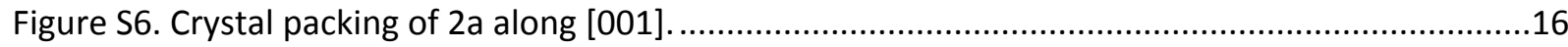

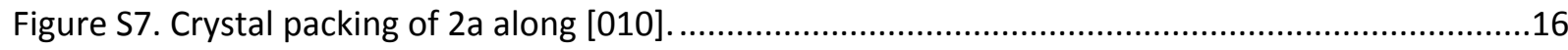

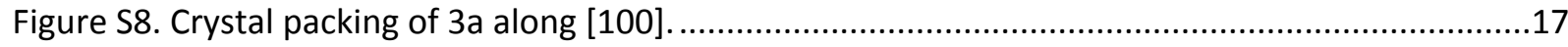

Figure S9. Comparison of complexes 2 and 3 XRPD patterns. Loosing of the crystallinity due to the $\mathrm{CH}_{3} \mathrm{CN}$ loss in both cases leads to widening of the diffraction peaks.............................................18

Figure S10. The topology of POM and $\left\{\mathrm{Ag}_{3}(\mathrm{DMA})_{6}\right\}^{3+}$ units connection in the crystal structure of $4 \ldots . .19$ Figure S11. The layer topology of crystal packing of 4. .19 
Figure S12. Comparison of experimental and calculated powder patterns for $5 \mathrm{a}$ and $5 \mathrm{~b}$. .20

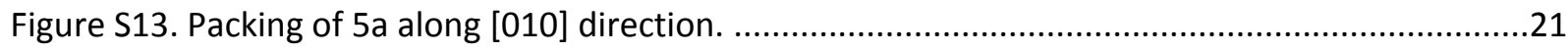



Figure S15. The tetrahedral orientation of POM anions around butterfly $\left\{\mathrm{Ag}_{4}(\mathrm{DEF})_{9}\right\}$ unit. ...............22

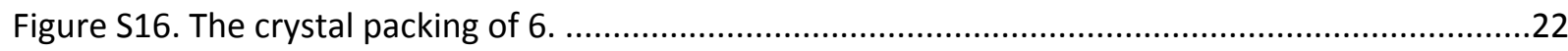

Figure S17. $\pi-\pi$ interactions between $\left[\left\{\mathrm{Ag}(\mathrm{PhCN})_{3}\right\}_{2} \mathrm{PW}_{11} \mathrm{NbO}_{40}\right]^{2-}$ in the crystal structure of $7 \ldots \ldots \ldots . . .23$



Figure S19. Kinetic of luminescence decay for powder $5 \mathrm{a} / 5 \mathrm{~b}$ at $77 \mathrm{~K}\left(\lambda_{\mathrm{Ex}}=390 \mathrm{~nm}, \lambda_{\mathrm{Em}}=535 \mathrm{~nm}\right) \ldots . .24$

Figure S20. Kinetics of luminescence decay for powder 7 at $77 \mathrm{~K}$ and $300 \mathrm{~K}\left(\lambda_{\mathrm{Ex}}=370 \mathrm{~nm}, \lambda_{\mathrm{Em}}=540\right.$



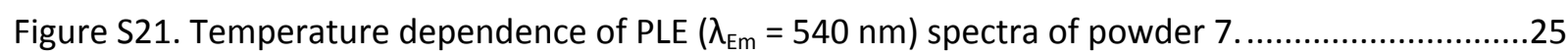




\section{Experimental Section}

General information

$\mathrm{AgNO}_{3}$, dimethylformamide (DMF), dimethylacetamide (DMA), diethylformamide (DEF) and N-Methyl-2-pyrrolidone (NMP) were of commercial quality (Sigma Aldrich) and were used as purchased. $\mathrm{H}_{4}\left[\mathrm{PW}_{11} \mathrm{NbO}_{40}\right] \cdot 8 \mathrm{H}_{2} \mathrm{O}$ was prepared according to standard ether method from $\mathrm{K}_{4}\left[\mathrm{PW}_{11} \mathrm{NbO}_{40}\right]$ [1]. IR ( $\left.v, \mathrm{~cm}^{-1}\right): 3102(\mathrm{w}), 700(\mathrm{w}), 1070(\mathrm{~m}), 963(\mathrm{~s}), 880(\mathrm{~m}), 740$ (vs), 589 (s). EDS calcd. Nb, W (\%): 3.2, 68,9. Found Nb, W (\%): 2.9, 70.7. TGA weight loss: 4.9\% ($\left.8 \mathrm{H}_{2} \mathrm{O} ; 30-300{ }^{\circ} \mathrm{C}\right) .{ }^{31} \mathrm{P}$ NMR $-13.2 \mathrm{ppm}$.

Elemental analysis was carried out on a Eurovector EA $3000 \mathrm{CHN}$ analyzer. FT-IR spectra were recorded on a FT-801 spectrometer (Simex, Russia). TGA experiments were done on a NETZSCH TG $209 \mathrm{~F} 1$ device in an Al crucible by heating a sample from 20 to $300{ }^{\circ} \mathrm{C}$ with $10^{\circ} \mathrm{C}$ gradient. The EDX was performed using a JSM 6700F scanning electron microscope with the EDS EX-23000 BU.

\section{Synthesis of $\left[\mathrm{Ag}_{4}(\mathrm{DMF})_{10}\right]\left[\mathrm{PW}_{11} \mathrm{NbO}_{40}\right](1)$ and $\left[\mathrm{Ag}_{4}(\mathrm{DMF})_{12}\right]\left[\mathrm{PW}_{11} \mathrm{NbO}_{40}\right]$ (1a):}

I. $0.100 \mathrm{~g}(0.034 \mathrm{mmol})$ of $\mathrm{H}_{4}\left[\mathrm{PW}_{11} \mathrm{NbO}_{40}\right] \cdot 8 \mathrm{H}_{2} \mathrm{O}$ was dissolved in $5 \mathrm{~mL}$ of DMF. After $0.025 \mathrm{~g}$ (0.147 mmol) $\mathrm{AgNO}_{3}$ was added to a clear solution. This mixture $\mathbf{A}$ was heated to $80{ }^{\circ} \mathrm{C}$ and stirred for 40 min until the solution became yellow. Crystals of 1a formed during slow vapor diffusion of diethyl ether into the initial solution. After drying in air 1a turned into $\mathbf{1}$.

II. The mixture A was stirred for 20 min without any heating and crystals of 1 formed during slow vapor diffusion of diethyl ether into the initial solution.

Yield of 1 in both methods was $0.125 \mathrm{~g}$ (92.3\% based on acid). The phase purity of 1 was confirmed with PXRD (Fig. S1). IR (v, $\left.\mathrm{cm}^{-1}\right)$ : 2923 (w), 2005 (w), 1620 (s), 1489 (m), 1428 (m), $1373(\mathrm{~m}), 1248$ (w), 1107 (w), 1063 (s), 962 (s), 882 (m), 758 (vs), 658 (s), 632 (s), 591 (s), 568 (s) . Anal. Calcd for $\mathrm{C}_{30} \mathrm{H}_{70} \mathrm{Ag}_{4} \mathrm{~N}_{10} \mathrm{NbO}_{50} \mathrm{PW}_{11} \mathrm{C}, \mathrm{H}, \mathrm{N}(\%)$ : 9.1, 1.8, 3.5. Found C, H, N (\%): 9.2, 1.8, 3.6.

Synthesis of $\mathbf{A g}_{4}\left[\mathbf{P W}_{\mathbf{1 1}} \mathbf{N b O}_{40}\right] \cdot 7.5 \mathbf{C H}_{3} \mathbf{C N}(2): 0.100 \mathrm{~g}(0.034 \mathrm{mmol})$ of $\mathrm{H}_{4}\left[\mathrm{PW}_{11} \mathrm{NbO}_{40}\right] \cdot 8 \mathrm{H}_{2} \mathrm{O}$ was dissolved in $5 \mathrm{~mL}$ of $\mathrm{MeCN}$. After $0.025 \mathrm{~g}(0.147 \mathrm{mmol}) \mathrm{AgNO}_{3}$ was added to a clear solution. This mixture was stirred for $60 \mathrm{~min}$. After this solution was transferred in ether atmosphere at $5{ }^{\circ} \mathrm{C}$. Crystals of $\left[\mathrm{Ag}\left(\mathrm{CH}_{3} \mathrm{CN}\right)_{4}\right]_{2}\left\{\left[\mathrm{Ag}\left(\mathrm{CH}_{3} \mathrm{CN}\right)_{3}\right]_{2}\left[\mathrm{PW}_{11} \mathrm{NbO}_{40}\right]\right\}$ (2a) were isolated during slow vapor diffusion of diethyl ether into solution. Drying in air leads to loosing a part of $\mathrm{CH}_{3} \mathrm{CN}$ molecules and final product can be formulated as $\mathrm{Ag}_{4}\left[\mathrm{PW}_{11} \mathrm{NbO}_{40}\right] \cdot 7.5 \mathrm{CH}_{3} \mathrm{CN}$

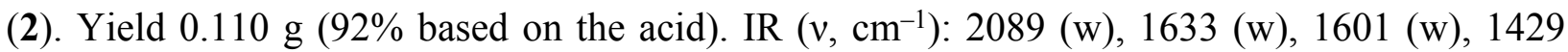
(w), 1369 (w), 1364 (w), 1062 (s), 960 (s), 881 (m), 738 (vs), 586 (vs), 568 (vs). Anal. Calcd for $\mathrm{C}_{15} \mathrm{H}_{22.5} \mathrm{Ag}_{4} \mathrm{~N}_{7.5} \mathrm{NbO}_{40} \mathrm{PW}_{11} \mathrm{C}, \mathrm{H}, \mathrm{N}(\%)$ : 5.1, 0.6, 3.0. Found C, H, N (\%): 5.0, 0.8, 3.0.

Synthesis of $\mathbf{A g}_{4}\left[\mathbf{P W}_{\mathbf{1 2}} \mathrm{O}_{\mathbf{4 0}}\right] \cdot 7.5 \mathbf{C H}_{3} \mathbf{C N}$ (3): $0.100 \mathrm{~g}(0.033 \mathrm{mmol})$ of $\mathrm{H}_{4}\left[\mathrm{SiW}_{12} \mathrm{O}_{40}\right] \cdot 7 \mathrm{H}_{2} \mathrm{O}$ was dissolved in $5 \mathrm{~mL}$ of $\mathrm{MeCN}$. After $0.025 \mathrm{~g}(0.147 \mathrm{mmol}) \mathrm{AgNO}_{3}$ was added to a clear solution. This mixture was stirred for $60 \mathrm{~min}$. Ether diffusion into reaction solution results in formation of $\left[\mathrm{Ag}\left(\mathrm{CH}_{3} \mathrm{CN}\right)_{3}\right]_{4}\left[\mathrm{SiW}_{12} \mathrm{O}_{40}\right]$ (3a) colorless crystals. Drying in air leads to loosing a part of $\mathrm{CH}_{3} \mathrm{CN}$ molecules and final product can be formulated as $\mathrm{Ag}_{4}\left[\mathrm{PW}_{12} \mathrm{O}_{40}\right] \cdot 7.5 \mathrm{CH}_{3} \mathrm{CN}$ (3). Yield $0.105 \mathrm{~g}\left(87.5 \%\right.$ based on acid). IR ( $\left.v, \mathrm{~cm}^{-1}\right): 2990(\mathrm{w}), 2928(\mathrm{w}), 1435(\mathrm{w}), 1401(\mathrm{w}), 1362(\mathrm{w})$, 1011 (w), 964 (m), 906 (s), 881 (vs), 875 (s), 729 (vs). Anal. Calcd for $\mathrm{C}_{15} \mathrm{H}_{22.5} \mathrm{Ag}_{4} \mathrm{~N}_{7.5} \mathrm{O}_{40} \mathrm{SiW}_{12}$ C, H, N (\%): 5.0, 0.6, 2.9. Found C, H, N (\%): 4.9 , 0.6, 2.9. 
Synthesis of $\left[\mathbf{A g}(\mathbf{D M A})_{4}\right]\left[\mathbf{A g}_{3}(\mathbf{D M A})_{6}\right]\left[\mathbf{P W}_{11} \mathbf{N b O}_{40}\right]$ (4): $0.100 \mathrm{~g}(0.028 \mathrm{mmol})$ of 2 was dissolved in $5 \mathrm{~mL}$ of dimethylacetamide. This mixture was heated for $80^{\circ} \mathrm{C}$ and stirred for 30 min. The crystals formed during slow vapor diffusion of diethyl ether into obtained solution. Yield $0.082 \mathrm{~g}\left(69.8 \%\right.$ based on 2). IR ( $\left.\mathrm{v}, \mathrm{cm}^{-1}\right): 2935(\mathrm{w}), 1628(\mathrm{sh}), 1600(\mathrm{~m}), 1578(\mathrm{~m}), 1503$ (w), 1454 (w), 1417 (w), 1395 (w), 1359 (w), 1259 (w), 1190 (w), $1080(w), 1066$ (m), 1015 (w), 960 (s), 886 (m), 776 (vs), 634 (m), 592 (s). Anal. Calcd for $\mathrm{C}_{40} \mathrm{H}_{90} \mathrm{Ag}_{4} \mathrm{~N}_{14} \mathrm{NbO}_{50} \mathrm{PW}_{11} \mathrm{C}, \mathrm{H}, \mathrm{N}$ (\%): 11.6, 2.2, 4.7. Found C, H, N (\%): 11.5, 2.2, 4.6.

\section{Synthesis of $\quad\left[\mathrm{Ag}_{2}(\mathrm{NMP})_{4}\left(\mathrm{CH}_{3} \mathrm{CN}\right)\right]_{2}\left[\mathrm{PW}_{11} \mathrm{NbO}_{40}\right] \cdot 1.3 \mathrm{NMP}$ $\left[\mathrm{Ag}_{2}(\mathrm{NMP})_{5}\right]_{2}\left[\mathrm{PW}_{11} \mathrm{NbO}_{40}\right](5 \mathrm{~b})$ :}

(5a)

and

$0.100 \mathrm{~g}(0.028 \mathrm{mmol})$ of $\mathrm{Ag}_{4}(\mathrm{MeCN})_{7.5}\left[\mathrm{PW}_{11} \mathrm{NbO}_{40}\right]$ was dissolved in $5 \mathrm{ml}$ of $\mathrm{N}$-methyl-2pyrrolidone. This mixture was heated for $80{ }^{\circ} \mathrm{C}$ and stirred for $60 \mathrm{~min}$. The crude product was obtained by slow vapor diffusion of diethyl ether into the solution. To get crystal product this solid was again dissolved in $2 \mathrm{ml}$ of $\mathrm{N}$-methyl-2-pyrrolidone and diffused by diethyl ether at 2 ${ }^{\circ} \mathrm{C}$. Yield $0.032 \mathrm{~g}\left(28.1 \%\right.$ based on $\left.\left\{\mathrm{PW}_{11} \mathrm{Nb}\right\}\right)$. IR (v, $\left.\mathrm{cm}^{-1}\right): 2960(\mathrm{w}), 2928(\mathrm{w}), 2876(\mathrm{w}), 1617$ (vs), 1501 (s), 1474 (m), 1401 (m), 1305 (m), 1259 (m), 1232 (w), 1177 (w), $1116(\mathrm{w}), 1065$ (w), 963 (vs), 883 (s), 785 (vs), 657 (s), 592 (s). Anal. Calcd for $\mathrm{C}_{50.5} \mathrm{H}_{89.1} \mathrm{Ag}_{4} \mathrm{~N}_{11.3} \mathrm{NbO}_{49.3} \mathrm{PW}_{11} \mathrm{C}, \mathrm{H}$, $\mathrm{N}(\%): 14.4,2.2,3.7$. Found C, H, N (\%): 14.5, 2.5, 3.5 .

\section{Synthesis of $\mathrm{Ag}_{4}\left[\mathrm{PW}_{11} \mathrm{NbO}_{40}\right] \cdot 9.5 \mathrm{DEF}(6)$ :}

$0.100 \mathrm{~g}(0.034 \mathrm{mmol})$ of $\mathrm{H}_{4}\left[\mathrm{PW}_{11} \mathrm{NbO}_{40}\right] \cdot 8 \mathrm{H}_{2} \mathrm{O}$ was dissolved in $5 \mathrm{ml}$ of diethylformamide. After $0.025 \mathrm{~g}(0.147 \mathrm{mmol}) \mathrm{AgNO}_{3}$ was added to a clear solution. This mixture was stirred for $60 \mathrm{~min}$. Crystals formed during slow vapor diffusion of diethyl ether into that solution. Yield $0.038 \mathrm{~g}$ (27\% based on $\left.\left\{\mathrm{PW}_{11} \mathrm{Nb}\right\}\right) . \mathrm{IR}\left(\mathrm{v}, \mathrm{cm}^{-1}\right): 2979(\mathrm{~m}), 2936(\mathrm{~m}), 2877(\mathrm{w}), 1613$ (vs), $1443(\mathrm{~s})$, 1390 (s), 1359 (s), 1305 (w), 1265 (m), 1208 (m), 1120 (m), 1104 (w), 1064 (vs), 959 (vs), 884 (s), 771 (vs), 639 (vs), 591 (s). Anal. Calcd for $\mathrm{C}_{47.5} \mathrm{H}_{104} \mathrm{Ag}_{4} \mathrm{~N}_{10} \mathrm{NbO}_{49.5} \mathrm{PW}_{11} \mathrm{C}, \mathrm{H}, \mathrm{N}$ (\%): 13.7, 2.5, 3.2. Found $\mathrm{C}, \mathrm{H}, \mathrm{N}(\%)$ : 13.7, 2.5, 3.2.

\section{Synthesis of $\left[\operatorname{Ag}(\mathrm{PhCN})_{4}\right]_{2}\left[\left\{\operatorname{Ag}(\mathrm{PhCN})_{3}\right\}_{2} \mathrm{PW}_{11} \mathrm{NbO}_{40}\right](7)$ :}

$0.100 \mathrm{~g}(0.034 \mathrm{mmol})$ of $\mathrm{H}_{4}\left[\mathrm{PW}_{11} \mathrm{NbO}_{40}\right] \cdot 8 \mathrm{H}_{2} \mathrm{O}$ was stirred in $5 \mathrm{ml}$ of PhCN. After $0.025 \mathrm{~g}(0.147$ mmol) $\mathrm{AgNO}_{3}$ was added to a white suspension. This solution became clear in 5 min, after it was stirred for $30 \mathrm{~min}$. Crystals formed during slow vapor diffusion of diethyl ether into that solution. Yield $0.114 \mathrm{~g}\left(72 \%\right.$ based on $\left.\left\{\mathrm{PW}_{11} \mathrm{Nb}\right\}\right)$. IR $\left(\mathrm{v}, \mathrm{cm}^{-1}\right): 1592(\mathrm{w}), 1486(\mathrm{w}), 1446(\mathrm{~m}), 1338$ (w), $1291(\mathrm{w}), 1197(\mathrm{w}), 1178$ (w), 1065 (s), 1025 (w), 956 (s), 885 (m), 844 (sh), 778 (vs), 749 (vs), 681 (vs), 623 (s), 592 (s). Anal. Calcd for $\mathrm{C}_{91} \mathrm{H}_{65} \mathrm{Ag}_{4} \mathrm{~N}_{13} \mathrm{NbO}_{40} \mathrm{PW}_{11} \mathrm{C}, \mathrm{H}, \mathrm{N}$ (\%): 24.0, 1.4 , 4.0. Found $\mathrm{C}, \mathrm{H}, \mathrm{N}(\%): 23.5,1.5,4.0$.

$X R D$. Crystallographic data and refinement details for 1, 1a, 2a, 3a, 4, 5a, 5b, 6, 7 are given in Table S1. The diffraction data were collected on a New Xcalibur (Agilent Technologies) diffractometer with MoK $\alpha$ radiation $(\lambda=0.71073)$ by doing $\varphi$ scans of wide $\left(1.0^{\circ}\right)$ frames at $130 \mathrm{~K}$. Absorption correction was done empirically using SCALE3 ABSPACK (CrysAlisPro, Agilent Technologies, Version 1.171.37.35 (release 13-08-2014 CrysAlis171 .NET)). Structures were solved by SHELXT[2] and refined by full-matrix least-squares treatment against $|\mathrm{F}|^{2}$ in anisotropic approximation with SHELXL 2017/1[3] in ShelXle program [4].

Typically, some residual electronic density around heavy elements due to high X-ray absorption is present. 
In the crystal structure of $\mathbf{3 a}\left\{\mathrm{Ag}\left(\mathrm{CH}_{3} \mathrm{CN}\right)_{3}\right\}^{+}$units are highly disordered, the dominant component is present.

In the crystal structure of complex 5a refinement gives several positions of highly disordered NMP molecules. SQUEEZE[5] procedure of PLATON[6] was used to estimate the number of NMP molecules of crystallization. According to the data $75 \mathrm{e}$ per $214 \AA^{3}$ were found which corresponds to c.a. 1.3 NMP molecules (54e, $\mathrm{Vm}=160 \AA^{3}$ for molecule).

$\mathrm{The} \mathrm{Nb} / \mathrm{W}$ statistical occupancies were refined based on typical EADP/EXYZ procedure. The information about Ag-O and Ag-N bond distances are summarized in Table S2.

The crystallographic data have been deposed with the Cambridge Crystallographic Data Centre under the deposition codes CCDC 1958862-1958870.

Luminescence. Corrected luminescence spectra were recorded on a Fluorolog 3 spectrometer (Horiba Jobin Yvon) with a cooled PC177CE-010 photon detection module equipped R2658 photomultiplier; with continuous $450 \mathrm{~W}$ and pulsed 50W Xe-lamps; with two Czerny-Turner double monochromators. Temperature dependences of luminescence were studied using Optistat DN optical cryostats (Oxford Instruments). Lifetime decay kinetic curves were recorded using 50W Xe-lamp $(\tau>>3 \mu \mathrm{s})$. Lifetime decay kinetic curves $(\tau<<10 \mu \mathrm{s})$ were recorded by TimeCorrelated Single Photon Counting (TCSPC) technique using NanoLED pulsed light sources and NanoLED-C2 controller. Analysis of TCSPC kinetics curves were carried out by standard program package DAS6 Analysis.

[1] P.A. Abramov, A.A. Shmakova, M. Haouas, G. Fink, E. Cadot, M.N. Sokolov, New J. Chem. 41 (2017) 256-262.

[2] G.M. Sheldrick, Acta Cryst. Sect. A Found. Adv. 71 (2015) 3-8.

[3] G.M. Sheldrick, Acta Cryst. Sect. C Struct. Chem. 71 (2015) 3-8.

[4] C.B. Hübschle, G.M. Sheldrick, B. Dittrich, J. Appl. Crystallogr. 44 (2011) 1281-1284.

[5] A.L. Spek, Acta Cryst. Sect. C Struct. Chem. 71 (2015) 9-18.

[6] A.L. Spek, Acta Cryst. 65 (2009) 148-155. 
Table S1. XRD Experimental details.

\begin{tabular}{|c|c|c|c|c|}
\hline & 1 & $1 \mathrm{a}$ & $2 a$ & $3 \mathbf{a}$ \\
\hline Chemical formula & $\begin{array}{l}\mathrm{C}_{30} \mathrm{H}_{70} \mathrm{Ag}_{4} \mathrm{~N}_{10} \mathrm{Nb} \\
\mathrm{O}_{50} \mathrm{PW}_{11}\end{array}$ & $\begin{array}{l}\mathrm{C}_{36} \mathrm{H}_{84} \mathrm{Ag}_{4} \mathrm{~N}_{12} \mathrm{Nb} \\
\mathrm{O}_{52} \mathrm{PW}_{11}\end{array}$ & $\begin{array}{l}\mathrm{C}_{28} \mathrm{H}_{42} \mathrm{Ag}_{4} \mathrm{~N}_{14} \mathrm{Nb} \\
\mathrm{O}_{40} \mathrm{PW}_{11}\end{array}$ & $\begin{array}{l}\mathrm{C}_{23} \mathrm{H}_{12} \mathrm{Ag}_{4} \mathrm{~N}_{12} \mathrm{O}_{40} \\
\mathrm{SiW}_{12}\end{array}$ \\
\hline$M_{\mathrm{r}}$ & 3948.67 & 4094.86 & 3792.46 & 3762.22 \\
\hline $\begin{array}{l}\text { Crystal system, space } \\
\text { group }\end{array}$ & Triclinic, $P^{-} 1$ & $\begin{array}{l}\text { Monoclinic, } \\
P 2_{1} / c\end{array}$ & $\begin{array}{l}\text { Monoclinic, } \\
P 2_{1} / c\end{array}$ & $\begin{array}{l}\text { Tetragonal, } \\
P 4_{1} 22\end{array}$ \\
\hline Temperature (K) & 130 & 130 & 130 & 130 \\
\hline$a, b, c(\AA)$ & $\begin{array}{l}12.0115(6), \\
12.7735(7), \\
13.5724(7)\end{array}$ & $\begin{array}{l}12.6651(6), \\
21.5069(10), \\
15.7472(8)\end{array}$ & $\begin{array}{l}24.140(2), \\
17.2713(11), \\
17.6494(15)\end{array}$ & $\begin{array}{l}12.3912(3) \\
12.3912(3) \\
45.7450(18)\end{array}$ \\
\hline$\alpha, \beta, \gamma\left(^{\circ}\right)$ & $\begin{array}{l}90.580(4), \\
98.755(4), \\
114.257(5)\end{array}$ & $\begin{array}{l}90,104.020(5), \\
90\end{array}$ & $\begin{array}{l}90,108.304(10), \\
90\end{array}$ & $90,90,90$ \\
\hline$V\left(\AA^{3}\right)$ & $1870.32(18)$ & $4161.6(4)$ & $6986.2(11)$ & $7023.8(4)$ \\
\hline$Z$ & 1 & 2 & 4 & 4 \\
\hline$\mu\left(\mathrm{mm}^{-1}\right)$ & 18.13 & 16.30 & 19.39 & 20.75 \\
\hline Crystal size (mm) & $\begin{array}{l}0.20 \times 0.10 \times \\
0.08\end{array}$ & $\begin{array}{l}0.30 \times 0.30 \times \\
0.20\end{array}$ & $\begin{array}{l}0.20 \times 0.15 \times \\
0.15\end{array}$ & $\begin{array}{l}0.12 \times 0.12 \times \\
0.08\end{array}$ \\
\hline$T_{\min }, T_{\max }$ & $0.565,1.000$ & $0.372,1.000$ & $0.390,1.000$ & $0.618,1.000$ \\
\hline $\begin{array}{l}\text { No. of measured, } \\
\text { independent and } \\
\text { observed }[I>2 \sigma(I)] \\
\text { reflections }\end{array}$ & $\begin{array}{l}15613,8683 \\
7339\end{array}$ & $\begin{array}{l}24020,9796 \\
8455\end{array}$ & $\begin{array}{l}41056,16564, \\
10273\end{array}$ & $\begin{array}{l}21470,8329 \\
6913\end{array}$ \\
\hline$R_{\text {int }}$ & 0.035 & 0.037 & 0.057 & 0.113 \\
\hline$\theta$ values $\left(^{\circ}\right)$ & $\begin{array}{l}\theta_{\max }=29.7, \theta_{\min } \\
=3.3\end{array}$ & $\begin{array}{l}\theta_{\max }=29.5, \theta_{\min } \\
=3.4\end{array}$ & $\begin{array}{l}\theta_{\max }=29.6, \theta_{\min } \\
=3.3\end{array}$ & $\begin{array}{l}\theta_{\max }=29.5, \theta_{\min } \\
=3.3\end{array}$ \\
\hline$(\sin \theta / \lambda)_{\max }\left(\AA^{-1}\right)$ & 0.697 & 0.694 & 0.694 & 0.694 \\
\hline Range of $h, k, l$ & $\begin{array}{l}-14 \leq h \leq 16 \\
-16 \leq k \leq 16 \\
-18 \leq l \leq 16\end{array}$ & $\begin{array}{l}-17 \leq h \leq 13 \\
-28 \leq k \leq 28 \\
-17 \leq l \leq 20\end{array}$ & $\begin{array}{l}-32 \leq h \leq 27 \\
-23 \leq k \leq 16 \\
-24 \leq l \leq 23\end{array}$ & $\begin{array}{l}-11 \leq h \leq 16 \\
-16 \leq k \leq 16 \\
-62 \leq l \leq 43\end{array}$ \\
\hline $\begin{array}{l}R\left[F^{2}>2 \sigma\left(F^{2}\right)\right] \\
w R\left(F^{2}\right), S\end{array}$ & $\begin{array}{l}0.045,0.101 \\
1.09\end{array}$ & $\begin{array}{l}0.056,0.107 \\
1.28\end{array}$ & $\begin{array}{l}0.085,0.158 \\
1.20\end{array}$ & $\begin{array}{l}0.100,0.204 \\
1.14\end{array}$ \\
\hline $\begin{array}{l}\text { No. of reflections, } \\
\text { parameters, restraints }\end{array}$ & $8683,462,31$ & $9796,504,0$ & $16564,831,96$ & $8329,275,0$ \\
\hline Weighting scheme & $\begin{array}{l}w=1 /\left[\sigma^{2}\left(F_{\mathrm{o}}^{2}\right)+\right. \\
(0.0321 P)^{2}+ \\
17.8215 P] \\
\text { where } P=\left(F_{\mathrm{o}}^{2}+\right. \\
\left.2 F_{\mathrm{c}}^{2}\right) / 3\end{array}$ & $\begin{array}{l}w=1 /\left[\sigma^{2}\left(F_{\mathrm{o}}^{2}\right)+\right. \\
125.1021 P] \\
\text { where } P=\left(F_{\mathrm{o}}^{2}+\right. \\
\left.2 F_{\mathrm{c}}^{2}\right) / 3\end{array}$ & $\begin{array}{l}w=1 /\left[\sigma^{2}\left(F_{\mathrm{o}}^{2}\right)+\right. \\
(0.0048 P)^{2}+ \\
440.4018 P] \\
\text { where } P=\left(F_{\mathrm{o}}^{2}+\right. \\
\left.2 F_{\mathrm{c}}^{2}\right) / 3\end{array}$ & $\begin{array}{l}w=1 /\left[\sigma^{2}\left(F_{\mathrm{o}}^{2}\right)+\right. \\
(0.0001 P)^{2}+ \\
1053.3436 P] \\
\text { where } P=\left(F_{\mathrm{o}}^{2}+\right. \\
\left.2 F_{\mathrm{c}}^{2}\right) / 3\end{array}$ \\
\hline$\Delta \rho_{\max }, \Delta \rho_{\min }\left(\mathrm{e} \AA^{-3}\right)$ & $2.24,-3.58$ & $1.79,-2.47$ & $2.42,-3.38$ & $3.43,-3.59$ \\
\hline Absolute structure & - & - & - & $\begin{array}{l}\text { Refined as an } \\
\text { inversion twin. }\end{array}$ \\
\hline $\begin{array}{l}\text { Absolute structure } \\
\text { parameter }\end{array}$ & - & - & - & $0.14(6)$ \\
\hline
\end{tabular}




\begin{tabular}{|c|c|c|c|c|}
\hline & 4 & $5 a$ & $5 b$ & 6 \\
\hline Chemical formula & \begin{tabular}{l|}
$\mathrm{C}_{40} \mathrm{H}_{90} \mathrm{Ag}_{4} \mathrm{~N}_{10} \mathrm{Nb}$ \\
$\mathrm{O}_{50} \mathrm{PW}_{11}$
\end{tabular} & $\begin{array}{l}\mathrm{C}_{44} \mathrm{H}_{78} \mathrm{Ag}_{4} \mathrm{~N}_{10} \mathrm{Nb} \\
\mathrm{O}_{48} \mathrm{PW}_{11}\end{array}$ & $\begin{array}{l}\mathrm{C}_{50} \mathrm{H}_{90} \mathrm{Ag}_{4} \mathrm{~N}_{10} \mathrm{Nb} \\
\mathrm{O}_{50} \mathrm{PW}_{11}\end{array}$ & $\begin{array}{l}\mathrm{C}_{45.50} \mathrm{H}_{97} \mathrm{Ag}_{4} \mathrm{~N}_{9.50} \\
\mathrm{NbO}_{49.50} \mathrm{PW}_{11}\end{array}$ \\
\hline$M_{\mathrm{r}}$ & 4088.92 & 4092.87 & 4209.02 & 4147.03 \\
\hline $\begin{array}{l}\text { Crystal system, space } \\
\text { group }\end{array}$ & $\begin{array}{l}\text { Monoclinic, } \\
P 2_{1} / n \\
\end{array}$ & Triclinic, $P^{-} 1$ & $\begin{array}{l}\text { Monoclinic, } \\
P 2_{1} / c\end{array}$ & Monoclinic, $C 2 / c$ \\
\hline Temperature (K) & 130 & 130 & 150 & 130 \\
\hline$a, b, c(\AA)$ & $\begin{array}{l}\text { 16.9188(6), } \\
17.7694(9), \\
29.1389(13)\end{array}$ & $\begin{array}{l}13.3560(5), \\
13.5403(6), \\
14.3473(6)\end{array}$ & \begin{tabular}{|l} 
13.1397(11), \\
$25.358(3)$ \\
$13.4363(9)$
\end{tabular} & $\begin{array}{l}17.0226(4), \\
22.4181(5), \\
48.5487(13)\end{array}$ \\
\hline$\alpha, \beta, \gamma\left(^{\circ}\right)$ & $\begin{array}{l}90,105.706(4), \\
90\end{array}$ & $\begin{array}{l}82.617(4), \\
67.742(4), \\
74.994(4)\end{array}$ & $\begin{array}{l}90,102.413(8), \\
90\end{array}$ & $\begin{array}{l}90,99.135(3), \\
90\end{array}$ \\
\hline$V\left(\AA^{3}\right)$ & $8433.2(7)$ & $2318.06(18)$ & $4372.3(7)$ & $18291.9(8)$ \\
\hline$Z$ & 4 & 1 & 2 & 8 \\
\hline$\mu\left(\mathrm{mm}^{-1}\right)$ & 16.09 & 14.63 & 15.52 & 14.83 \\
\hline Crystal size (mm) & $\begin{array}{l}0.25 \times 0.20 \times \\
0.06\end{array}$ & $\begin{array}{l}0.22 \times 0.16 \times \\
0.12\end{array}$ & $\begin{array}{l}0.25 \times 0.12 \times \\
0.03\end{array}$ & $\begin{array}{l}0.26 \times 0.18 \times \\
0.08\end{array}$ \\
\hline$T_{\min }, T_{\max }$ & $0.093,1.000$ & $0.467,1.000$ & $0.413,1.000$ & $0.123,1.000$ \\
\hline $\begin{array}{l}\text { No. of measured, } \\
\text { independent and } \\
\text { observed }[I>2 \sigma(I)] \\
\text { reflections }\end{array}$ & $\begin{array}{l}47034,19802, \\
12214\end{array}$ & $\begin{array}{l}20666,10776, \\
8952\end{array}$ & $\begin{array}{l}25282,10418, \\
6199\end{array}$ & $\begin{array}{l}40714,20982, \\
14708\end{array}$ \\
\hline$R_{\text {int }}$ & 0.090 & 0.028 & 0.053 & 0.049 \\
\hline$\theta$ values $\left(^{\circ}\right)$ & $\begin{array}{l}\theta_{\max }=29.5, \theta_{\min } \\
=3.3\end{array}$ & $\begin{array}{l}\theta_{\max }=29.5, \theta_{\min } \\
=3.4\end{array}$ & $\begin{array}{l}\theta_{\max }=29.6, \theta_{\min } \\
=2.1\end{array}$ & $\begin{array}{l}\theta_{\max }=29.2, \theta_{\min } \\
=3.3\end{array}$ \\
\hline$(\sin \theta / \lambda)_{\max }\left(\AA^{-1}\right)$ & 0.693 & 0.693 & 0.695 & 0.686 \\
\hline Range of $h, k, l$ & $\begin{array}{l}-21 \leq h \leq 22 \\
-23 \leq k \leq 21 \\
-37 \leq l \leq 36\end{array}$ & $\begin{array}{l}-17 \leq h \leq 16 \\
-18 \leq k \leq 18 \\
-16 \leq l \leq 19\end{array}$ & $\begin{array}{l}-15 \leq h \leq 18 \\
-20 \leq k \leq 33 \\
-18 \leq l \leq 17\end{array}$ & $\begin{array}{l}-22 \leq h \leq 21 \\
-30 \leq k \leq 15 \\
-40 \leq l \leq 66\end{array}$ \\
\hline $\begin{array}{l}R\left[F^{2}>2 \sigma\left(F^{2}\right)\right] \\
w R\left(F^{2}\right), S\end{array}$ & $\begin{array}{l}0.076,0.189, \\
1.03\end{array}$ & $\begin{array}{l}0.041,0.089 \\
1.09\end{array}$ & $\begin{array}{l}0.064,0.171, \\
1.02\end{array}$ & $\begin{array}{l}0.064,0.141, \\
1.04\end{array}$ \\
\hline $\begin{array}{l}\text { No. of reflections, } \\
\text { parameters, restraints }\end{array}$ & $19802,1022,151$ & $10776,505,0$ & $10418,530,88$ & $20982,1050,38$ \\
\hline Weighting scheme & \begin{tabular}{|l|}
$w=1 /\left[\sigma^{2}\left(F_{\mathrm{o}}^{2}\right)+\right.$ \\
$(0.0535 P)^{2}+$ \\
$378.441 P]$ \\
where $P=\left(F_{\mathrm{o}}^{2}+\right.$ \\
$\left.2 F_{\mathrm{c}}^{2}\right) / 3$
\end{tabular} & $\begin{array}{l}w=1 /\left[\sigma^{2}\left(F_{\mathrm{o}}^{2}\right)+\right. \\
(0.0262 P)^{2}+ \\
16.6087 P] \\
\text { where } P=\left(F_{\mathrm{o}}^{2}+\right. \\
\left.2 F_{\mathrm{c}}^{2}\right) / 3\end{array}$ & \begin{tabular}{|l|}
$w=1 /\left[\sigma^{2}\left(F_{\mathrm{o}}^{2}\right)+\right.$ \\
$(0.0618 P)^{2}+$ \\
$70.131 P]$ \\
where $P=\left(F_{\mathrm{o}}^{2}+\right.$ \\
$\left.2 F_{\mathrm{c}}^{2}\right) / 3$
\end{tabular} & $\begin{array}{l}w=1 /\left[\sigma^{2}\left(F_{\mathrm{o}}^{2}\right)+\right. \\
(0.0441 P)^{2}+ \\
354.0696 P] \\
\text { where } P=\left(F_{\mathrm{o}}{ }^{2}+\right. \\
\left.2 F_{\mathrm{c}}^{2}\right) / 3\end{array}$ \\
\hline$\Delta \rho_{\max }, \Delta \rho_{\min }\left(\mathrm{e} \AA^{-3}\right)$ & $3.39,-3.63$ & $3.04,-4.29$ & $2.61,-2.40$ & $2.90,-4.34$ \\
\hline
\end{tabular}




\begin{tabular}{|l|l|}
\hline & 7 \\
\hline Chemical formula & $\mathrm{C}_{98} \mathrm{H}_{70} \mathrm{Ag}_{4} \mathrm{~N}_{14} \mathrm{NbO}_{40} \mathrm{PW}_{11}$ \\
\hline$M_{\mathrm{r}}$ & 4661.39 \\
\hline $\begin{array}{l}\text { Crystal system, space } \\
\text { group }\end{array}$ & Monoclinic, $C 2 / c$ \\
\hline Temperature $(\mathrm{K})$ & 130 \\
\hline$a, b, c(\AA)$ & $28.2977(10), 15.6032(4), 28.9550(12)$ \\
\hline$\alpha, \beta, \gamma\left(^{\circ}\right)$ & $90,114.989(5), 90$ \\
\hline$V\left(\AA^{3}\right)$ & $11587.8(8)$ \\
\hline$Z$ & 4 \\
\hline$\left.\mu(\mathrm{mm})^{-1}\right)$ & 11.72 \\
\hline Crystal size $(\mathrm{mm})$ & $0.12 \times 0.08 \times 0.06$ \\
\hline$T_{\min }, T_{\max }$ & $0.611,1.000$ \\
\hline $\begin{array}{l}\text { No. of measured, } \\
\text { independent and } \\
\text { observed }[I>2 \sigma(I)] \\
\text { reflections }\end{array}$ & $53140,14336,12023$ \\
\hline$R_{\text {int }}$ & 0.034 \\
\hline$\theta$ values $\left({ }^{\circ}\right)$ & $\theta_{\max }=29.6, \theta_{\min }=3.3$ \\
\hline$(\text { sin } \theta / \lambda)_{\max }\left(\AA^{-1}\right)$ & 0.695 \\
\hline Range of $h, k, l$ & $-38 \leq h \leq 32,-20 \leq k \leq 20,-27 \leq l \leq 39$ \\
\hline $\begin{array}{l}R\left[F^{2}>2 \sigma\left(F^{2}\right)\right], \\
w R\left(F^{2}\right), S\end{array}$ & $0.031,0.061,1.03$ \\
\hline $\begin{array}{l}\text { No. of reflections, } \\
\text { parameters, restraints }\end{array}$ & $14336,724,0$ \\
\hline $\begin{array}{l}\text { Weighting scheme } \\
\Delta \rho_{\max }, \Delta \rho_{\min }\left(\mathrm{e} \AA \AA^{-3}\right)\end{array}$ & $\begin{array}{l}w=1 /\left[\sigma^{2}\left(F_{\mathrm{o}}^{2}\right)+(0.0183 P)^{2}+89.6848 P\right] \\
\text { where } P=\left(F_{\mathrm{o}}^{2}+2 F_{\mathrm{c}}^{2}\right) / 3\end{array}$ \\
\hline
\end{tabular}

Computer programs: CrysAlis PRO 1.171.38.41 (Rigaku OD, 2015), SHELXS2014 (Sheldrick, 2014), SHELXL2014 (Sheldrick, 2014), ShelXle (Hübschle, 2011), CIFTAB-2014 (Sheldrick, 2014). 
Table S2. Selected geometric parameters $(\AA)$

\begin{tabular}{|c|c|c|c|}
\hline \multicolumn{4}{|c|}{1} \\
\hline $\mathrm{O} 23-\mathrm{Ag} 1$ & $2.412(8)$ & $\mathrm{O} 28-\mathrm{Ag} 2$ & $2.538(8)$ \\
\hline $\mathrm{O} 23-\mathrm{Ag} 2^{\mathrm{i}}$ & $2.587(8)$ & $\mathrm{O} 28-\mathrm{Ag} 2^{\mathrm{i}}$ & $2.581(8)$ \\
\hline $\mathrm{O} 24-\mathrm{Ag} 1$ & $2.393(9)$ & Ag1-O7B & $2.288(15)$ \\
\hline $\mathrm{O} 24-\mathrm{Ag} 2$ & $2.529(9)$ & Ag1-O7A & 2.475 (17) \\
\hline $\mathrm{O} 26-\mathrm{Ag} 2$ & $2.405(10)$ & $\mathrm{Ag} 1-\mathrm{O} 10 \mathrm{~B}^{\mathrm{ii}}$ & $2.49(3)$ \\
\hline $\mathrm{O} 27-\mathrm{Ag} 2$ & $2.380(9)$ & $\mathrm{Ag} 1-\mathrm{O}_{10 \mathrm{~A}^{\mathrm{ii}}}$ & $2.33(2)$ \\
\hline $\mathrm{O} 28-\mathrm{Ag} 1$ & $2.414(7)$ & & \\
\hline \multicolumn{4}{|c|}{$1 \mathrm{a}$} \\
\hline Ag1-Ag2 & $3.2158(16)$ & $\mathrm{O} 21-\mathrm{Ag} 2^{\mathrm{iii}}$ & $2.442(10)$ \\
\hline $\mathrm{Ag} 1-\mathrm{Ag} 2^{\mathrm{iii}}$ & $3.3704(17)$ & $\mathrm{O} 22-\mathrm{Ag} 2$ & $2.448(12)$ \\
\hline O19-Ag1 & $2.518(11)$ & $\mathrm{O} 23-\mathrm{Ag} 2$ & $2.314(11)$ \\
\hline $\mathrm{O} 20-\mathrm{Ag} 1$ & $2.389(10)$ & $\mathrm{O} 28-\mathrm{Ag} 1$ & $2.503(11)$ \\
\hline $\mathrm{O} 20-\mathrm{Ag} 2$ & $2.395(10)$ & $\mathrm{Ag} 1-\mathrm{O} 18 \mathrm{~B}$ & $2.429(19)$ \\
\hline $\mathrm{O} 21-\mathrm{Ag} 1$ & $2.340(11)$ & $\mathrm{Ag} 1-\mathrm{O} 18 \mathrm{~A}$ & $2.52(2)$ \\
\hline \multicolumn{4}{|c|}{$2 \mathbf{a}$} \\
\hline $\mathrm{Ag} 1 \mathrm{~A}-\mathrm{Ag} 1 \mathrm{~B}$ & $0.936(10)$ & $\mathrm{N} 12-\mathrm{Ag} 1 \mathrm{~A}$ & $2.46(4)$ \\
\hline N1-Ag3 & $2.209(19)$ & Ag1B-N11B & $2.28(7)$ \\
\hline $\mathrm{N} 2-\mathrm{Ag} 3$ & $2.44(2)$ & Ag1A-N11B & $1.82(7)$ \\
\hline $\mathrm{N} 3-\mathrm{Ag} 3$ & $2.34(2)$ & Ag1A-N11A & $2.24(4)$ \\
\hline $\mathrm{N} 4-\mathrm{Ag} 3$ & $2.27(2)$ & $\mathrm{Ag} 2 \mathrm{~B}-\mathrm{N} 8$ & $1.99(4)$ \\
\hline N5-Ag4B & $2.20(3)$ & Ag2B-N35 & $2.17(5)$ \\
\hline $\mathrm{N} 5-\mathrm{Ag} 4 \mathrm{~A}$ & $2.22(3)$ & $\mathrm{Ag} 2 \mathrm{~A}-\mathrm{N} 18$ & $2.49(6)$ \\
\hline N7-Ag2B & $2.26(4)$ & $\mathrm{Ag} 2 \mathrm{~A}-\mathrm{N} 21$ & $2.13(5)$ \\
\hline $\mathrm{N} 7-\mathrm{Ag} 2 \mathrm{~A}$ & $2.27(3)$ & Ag4B-N6 & $2.55(5)$ \\
\hline N9-Ag1B & $2.30(3)$ & Ag4B-N19A & $1.97(5)$ \\
\hline N9-Ag1A & $2.23(3)$ & Ag4B-N19 & $2.19(7)$ \\
\hline N10B-Ag1B & $1.86(4)$ & Ag4B-N38 & $2.29(6)$ \\
\hline $\mathrm{N} 10 \mathrm{~B}-\mathrm{Ag} 1 \mathrm{~A}$ & $2.32(4)$ & $\mathrm{Ag} 4 \mathrm{~A}-\mathrm{N} 6$ & $2.25(5)$ \\
\hline N10A-Ag1B & $2.06(10)$ & Ag4A-N19A & $2.23(5)$ \\
\hline N12-Ag1B & $2.10(3)$ & Ag4A-N19 & $2.33(7)$ \\
\hline \multicolumn{4}{|c|}{$3 a$} \\
\hline $\mathrm{Ag} 1-\mathrm{N} 1 \mathrm{~A}$ & $2.34(5)$ & Ag3-N9 & $2.48(8)$ \\
\hline Ag1-N21 & $2.01(7)$ & Ag3-N10 & $2.06(6)$ \\
\hline Ag2-N1B & $1.92(5)$ & Ag4-N5 & $2.22(6)$ \\
\hline $\mathrm{Ag} 2-\mathrm{N} 2$ & $2.33(10)$ & $\mathrm{Ag} 4-\mathrm{N} 6$ & $2.13(10)$ \\
\hline $\mathrm{Ag} 2-\mathrm{N} 3$ & $1.85(15)$ & Ag4-N7 & $2.18(12)$ \\
\hline
\end{tabular}




\begin{tabular}{|c|c|c|c|}
\hline $\mathrm{Ag} 3-\mathrm{N} 8$ & $2.13(8)$ & & \\
\hline \multicolumn{4}{|c|}{4} \\
\hline $\mathrm{O} 12-\mathrm{Ag} 2$ & $2.381(14)$ & $\mathrm{O} 46-\mathrm{Ag} 2$ & $2.455(16)$ \\
\hline $\mathrm{O} 16-\mathrm{Ag} 2^{\mathrm{iv}}$ & $2.457(13)$ & $\mathrm{O} 46-\mathrm{Ag} 3^{\mathrm{vii}}$ & $2.303(16)$ \\
\hline $\mathrm{O} 21-\mathrm{Ag}^{\mathrm{v}}$ & $2.468(14)$ & $\mathrm{O} 47-\mathrm{Ag} 4$ & $2.26(2)$ \\
\hline $\mathrm{O} 37-\mathrm{Ag} 3$ & $2.306(13)$ & $\mathrm{O} 48-\mathrm{Ag} 4$ & $2.317(19)$ \\
\hline $\mathrm{O} 41-\mathrm{Ag} 1$ & $2.500(13)$ & $\mathrm{O} 49-\mathrm{Ag} 4$ & $2.24(2)$ \\
\hline $\mathrm{O} 41-\mathrm{Ag} 2$ & $2.451(14)$ & $\mathrm{O} 50-\mathrm{Ag} 4$ & $2.249(19)$ \\
\hline $\mathrm{O} 43-\mathrm{Ag} 1$ & $2.454(16)$ & $\mathrm{Ag} 1-\mathrm{O} 42 \mathrm{~B}$ & $2.34(3)$ \\
\hline $\mathrm{O} 43-\mathrm{Ag} 2$ & $2.355(14)$ & $\mathrm{Ag} 1-\mathrm{O} 42 \mathrm{~A}$ & $2.50(6)$ \\
\hline $\mathrm{O} 45-\mathrm{Ag} 1^{\mathrm{vi}}$ & $2.454(16)$ & Ag1-O51 & $2.44(3)$ \\
\hline $\mathrm{O} 45-\mathrm{Ag} 3$ & $2.321(17)$ & $\mathrm{Ag} 1-\mathrm{O} 52$ & $2.32(6)$ \\
\hline \multicolumn{4}{|c|}{$5 a$} \\
\hline $\mathrm{Ag} 1-\mathrm{N} 4 \mathrm{~B}$ & $2.277(17)$ & $\mathrm{O} 20-\mathrm{Ag} 2$ & $2.509(5)$ \\
\hline $\mathrm{Ag} 1-\mathrm{N} 4 \mathrm{~A}$ & $2.24(3)$ & $\mathrm{O} 21-\mathrm{Ag} 1$ & $2.522(7)$ \\
\hline $\mathrm{O} 1-\mathrm{Ag} 2$ & $2.344(6)$ & $\mathrm{O} 21-\mathrm{Ag} 2$ & $2.340(7)$ \\
\hline $\mathrm{O} 19-\mathrm{Ag} 1$ & $2.324(6)$ & $\mathrm{O} 22-\mathrm{Ag} 2$ & $2.333(6)$ \\
\hline $\mathrm{O} 20-\mathrm{Ag} 1$ & $2.336(6)$ & $\mathrm{O} 22-\mathrm{Ag} 2^{\mathrm{iii}}$ & $2.532(6)$ \\
\hline \multicolumn{4}{|c|}{$5 \mathbf{b}$} \\
\hline O00G-Ag1 & $2.358(11)$ & $\mathrm{Ag} 2-\mathrm{O} 20 \mathrm{X}$ & $2.24(4)$ \\
\hline $\mathrm{O} 00 \mathrm{G}-\mathrm{Ag} 1^{\mathrm{ii}}$ & $2.444(13)$ & $\mathrm{Ag} 2-\mathrm{O} 20 \mathrm{~B}$ & $2.43(3)$ \\
\hline $\mathrm{O} 1-\mathrm{Ag} 1$ & $2.386(10)$ & $\mathrm{Ag} 2-\mathrm{O} 20 \mathrm{~A}$ & $2.43(2)$ \\
\hline O19-Ag1 & $2.511(14)$ & $\mathrm{Ag} 2-\mathrm{O} 21 \mathrm{~B}$ & $2.12(5)$ \\
\hline $\mathrm{O} 19-\mathrm{Ag} 2$ & $2.372(14)$ & $\mathrm{Ag} 2-\mathrm{O} 21 \mathrm{~A}$ & $2.20(2)$ \\
\hline $\mathrm{Ag} 1-\mathrm{O} 20 \mathrm{~B}$ & $2.40(3)$ & Ag2-O26X & $2.31(4)$ \\
\hline $\mathrm{Ag} 1-\mathrm{O} 20 \mathrm{~A}$ & $2.42(2)$ & & \\
\hline \multicolumn{4}{|c|}{6} \\
\hline $\mathrm{Ag} 3 \mathrm{~B}-\mathrm{Ag} 4 \mathrm{~B}$ & $2.690(5)$ & $\mathrm{O} 41-\mathrm{Ag} 4^{\mathrm{viii}}$ & $2.55(2)$ \\
\hline $\mathrm{Ag} 3 \mathrm{~B}-\mathrm{Ag} 4 \mathrm{~B}^{\text {viii }}$ & $3.113(5)$ & $\mathrm{O} 42-\mathrm{Ag} 3 \mathrm{~B}$ & $2.432(14)$ \\
\hline $\mathrm{Ag} 3 \mathrm{~B}-\mathrm{Ag} 4 \mathrm{~A}^{\text {viii }}$ & $2.894(5)$ & $\mathrm{O} 42-\mathrm{Ag} 4 \mathrm{~B}$ & $2.215(18)$ \\
\hline $\mathrm{Ag} 3 \mathrm{~B}-\mathrm{Ag} 4 \mathrm{~A}$ & $3.315(6)$ & $\mathrm{O} 42-\mathrm{Ag} 4$ & $1.70(2)$ \\
\hline $\mathrm{Ag} 3 \mathrm{~B}-\mathrm{Ag} 4$ & $1.83(2)$ & O43-Ag4B & $2.345(17)$ \\
\hline $\mathrm{Ag} 3 \mathrm{~A}-\mathrm{Ag} 4 \mathrm{~B}$ & $3.161(6)$ & $\mathrm{O} 43-\mathrm{Ag} 4 \mathrm{~A}$ & $2.061(17)$ \\
\hline $\mathrm{Ag} 3 \mathrm{~A}-\mathrm{Ag} 4 \mathrm{~B}^{\text {viii }}$ & $3.261(5)$ & O44-Ag3B & $2.492(18)$ \\
\hline $\mathrm{Ag} 3 \mathrm{~A}-\mathrm{Ag} 4 \mathrm{~A}^{\text {viii }}$ & $2.933(6)$ & $\mathrm{O} 44-\mathrm{Ag} 3 \mathrm{~A}$ & $2.100(18)$ \\
\hline $\mathrm{Ag} 3 \mathrm{~A}-\mathrm{Ag} 4$ & $2.29(2)$ & O45-Ag1 & $2.386(10)$ \\
\hline $\mathrm{Ag} 4 \mathrm{~B}-\mathrm{Ag} 4$ & $0.92(2)$ & $\mathrm{O} 45-\mathrm{Ag} 2^{\mathrm{ix}}$ & $2.429(10)$ \\
\hline
\end{tabular}




\begin{tabular}{|l|l|l|l|}
\hline $\mathrm{Ag} 4 \mathrm{~A}-\mathrm{Ag} 4 \mathrm{~B}$ & $0.746(5)$ & $\mathrm{O} 46-\mathrm{Ag} 1^{\mathrm{x}}$ & $2.438(10)$ \\
\hline $\mathrm{Ag} 4 \mathrm{~A}-\mathrm{Ag} 4$ & $1.60(2)$ & $\mathrm{O} 46-\mathrm{Ag} 1$ & $2.438(10)$ \\
\hline $\mathrm{O} 9-\mathrm{Ag} 2$ & $2.372(9)$ & $\mathrm{O} 47-\mathrm{Ag} 1$ & $2.428(11)$ \\
\hline $\mathrm{O} 17-\mathrm{Ag} 3 \mathrm{~B}$ & $2.403(10)$ & $\mathrm{O} 47-\mathrm{Ag} 2^{\mathrm{xi}}$ & $2.485(13)$ \\
\hline $\mathrm{O} 17-\mathrm{Ag} 3 \mathrm{~A}$ & $2.311(10)$ & $\mathrm{O} 48-\mathrm{Ag} 2$ & $2.336(11)$ \\
\hline $\mathrm{O} 36-\mathrm{Ag} 1$ & $2.363(11)$ & $\mathrm{O} 49-\mathrm{Ag} 1^{\mathrm{xii}}$ & $2.568(12)$ \\
\hline $\mathrm{O} 41-\mathrm{Ag} 3 \mathrm{~B}$ & $2.463(15)$ & $\mathrm{O} 49-\mathrm{Ag} 2$ & $2.427(10)$ \\
\hline $\mathrm{O} 41-\mathrm{Ag} 3 \mathrm{~B}^{\text {viii }}$ & $2.545(14)$ & $\mathrm{Ag} 4 \mathrm{~B}-\mathrm{O} 50 \mathrm{~B}$ & $2.32(3)$ \\
\hline $\mathrm{O} 41-\mathrm{Ag} 4 \mathrm{~B}$ & $2.437(12)$ & $\mathrm{Ag} 4 \mathrm{~A}-\mathrm{O} 50 \mathrm{~B}$ & $2.16(3)$ \\
\hline $\mathrm{O} 41-\mathrm{Ag} 4 \mathrm{~A}$ & $2.495(12)$ & $\mathrm{Ag} 4 \mathrm{~A}-\mathrm{O} 50 \mathrm{~A}$ & $2.35(4)$ \\
\hline $\mathrm{O} 41-\mathrm{Ag} 4$ & $2.23(2)$ & $\mathrm{Ag} 4-\mathrm{O} 50 \mathrm{~B}$ & $2.57(4)$ \\
\hline & & \multicolumn{2}{l}{} \\
\hline \multicolumn{2}{|l|}{} & $\mathbf{N} 5-\mathrm{Ag} 1$ & $2.497(5)$ \\
\hline $\mathrm{N} 1-\mathrm{Ag} 2$ & $2.249(6)$ & $\mathrm{N} 6-\mathrm{Ag} 1$ & $2.148(5)$ \\
\hline $\mathrm{N} 2-\mathrm{Ag} 2$ & $2.311(6)$ & $\mathrm{N} 7-\mathrm{Ag} 1$ & $2.225(6)$ \\
\hline $\mathrm{N} 3-\mathrm{Ag} 2$ & $2.205(5)$ & $\mathrm{O} 1-\mathrm{Ag} 1$ & $2.355(4)$ \\
\hline $\mathrm{N} 4-\mathrm{Ag} 2$ & $2.339(6)$ &
\end{tabular}

Symmetry code(s): (i) $-x+1,-y+1,-z$; (ii) $-x+1,-y+1,-z+1$; (iii) $-x+2,-y+1,-z+1$; (iv) $-x+3 / 2, y+1 / 2,-z+3 / 2$; (v) $x+1 / 2, y-1 / 2,-z+3 / 2$; (vi) $x-1, y, z$; (vii) $x+1, y, z$; (viii) $-x+3 / 2,-y+1 / 2,-z+1$; (ix) $x-1 / 2, y-1 / 2, z$; (x) $-x, y,-z+1 / 2$; (xi) $-x+1 / 2, y-1 / 2,-z+1 / 2$; (xii) $-x+1 / 2, y+1 / 2,-z+1 / 2$. 


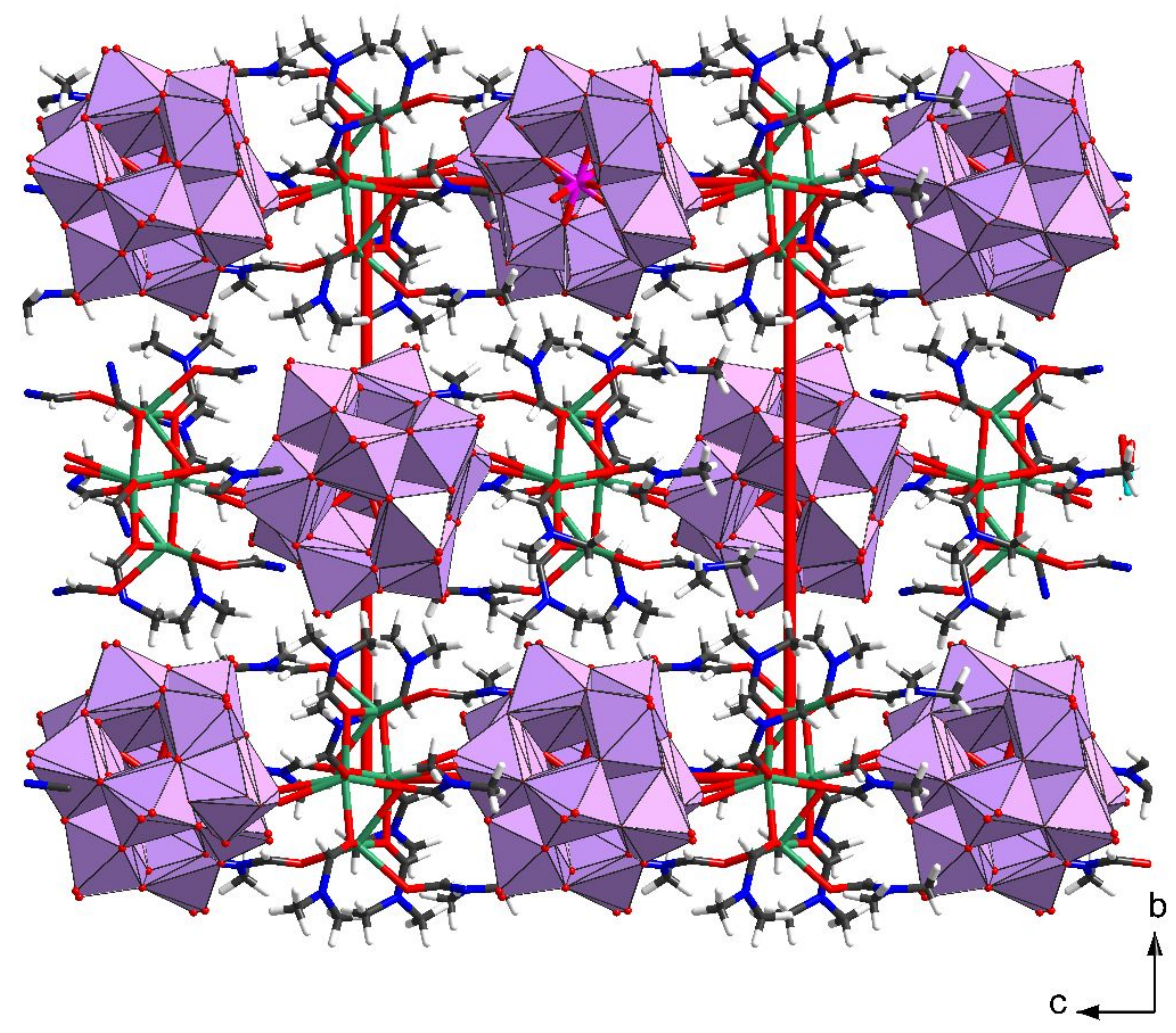

Figure S1. The crystal packing of 1a. 


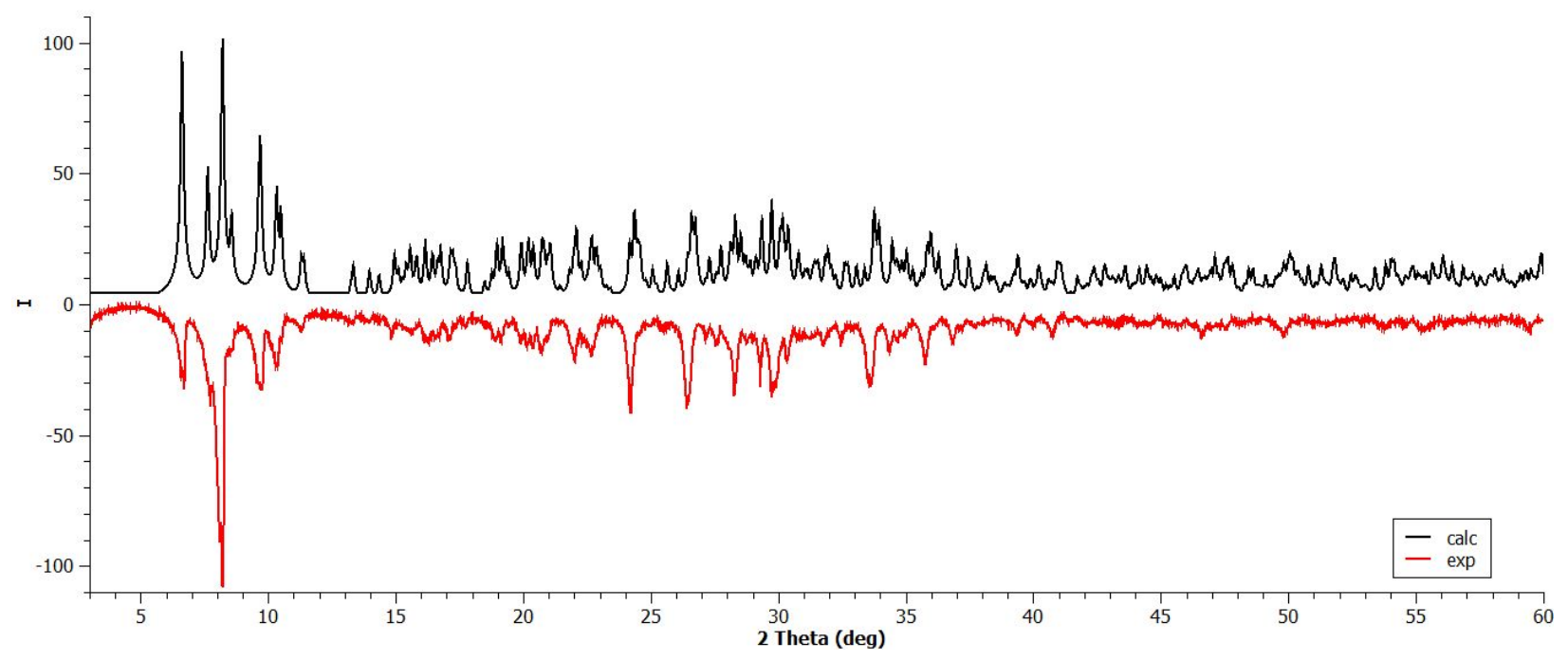

Figure S2. Comparison of observed and calculated PXRD patterns for 1. 


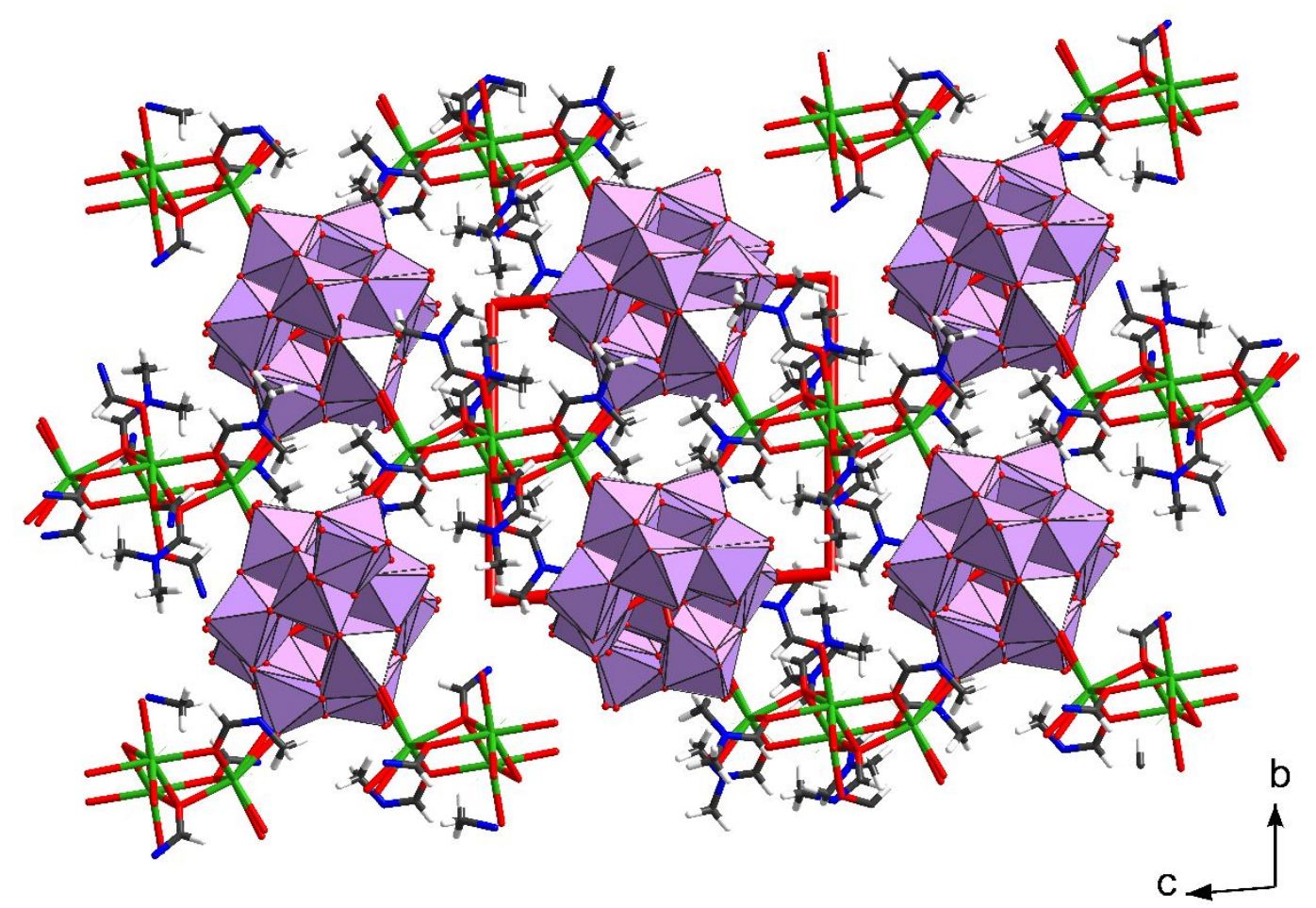

Figure S3. Crystal packing of 1 along [100] crystal direction.

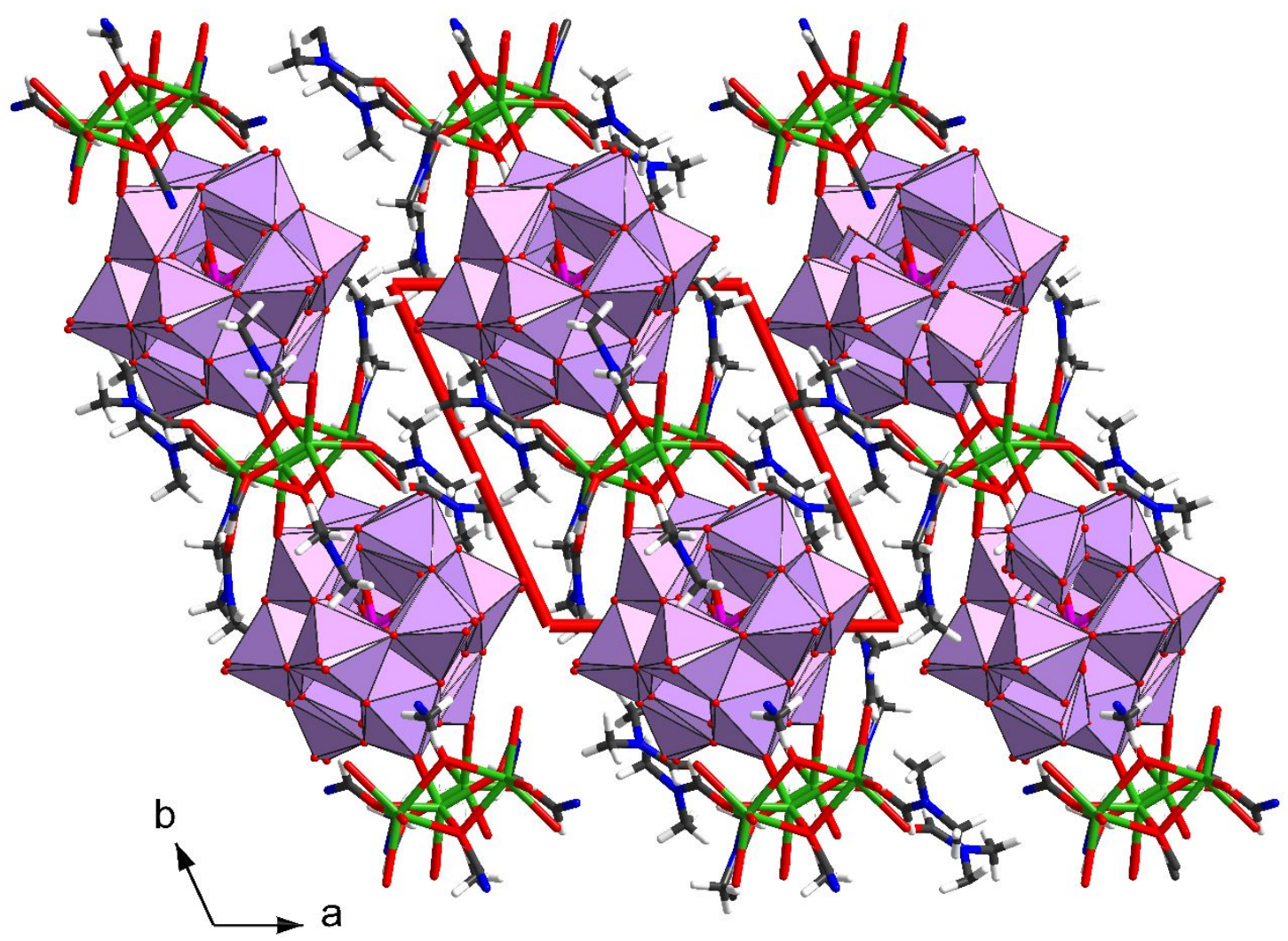

Figure S4. Layered crystal packing of 1 along [001]. 




Figure S5. The UV-VIS spectrum of 1a in DMF. The absorption band at $267 \mathrm{~nm}$ overlaps with absorption of DMF which gives incorrect profile of the band. 




Figure S6. Crystal packing of 2a along [001].

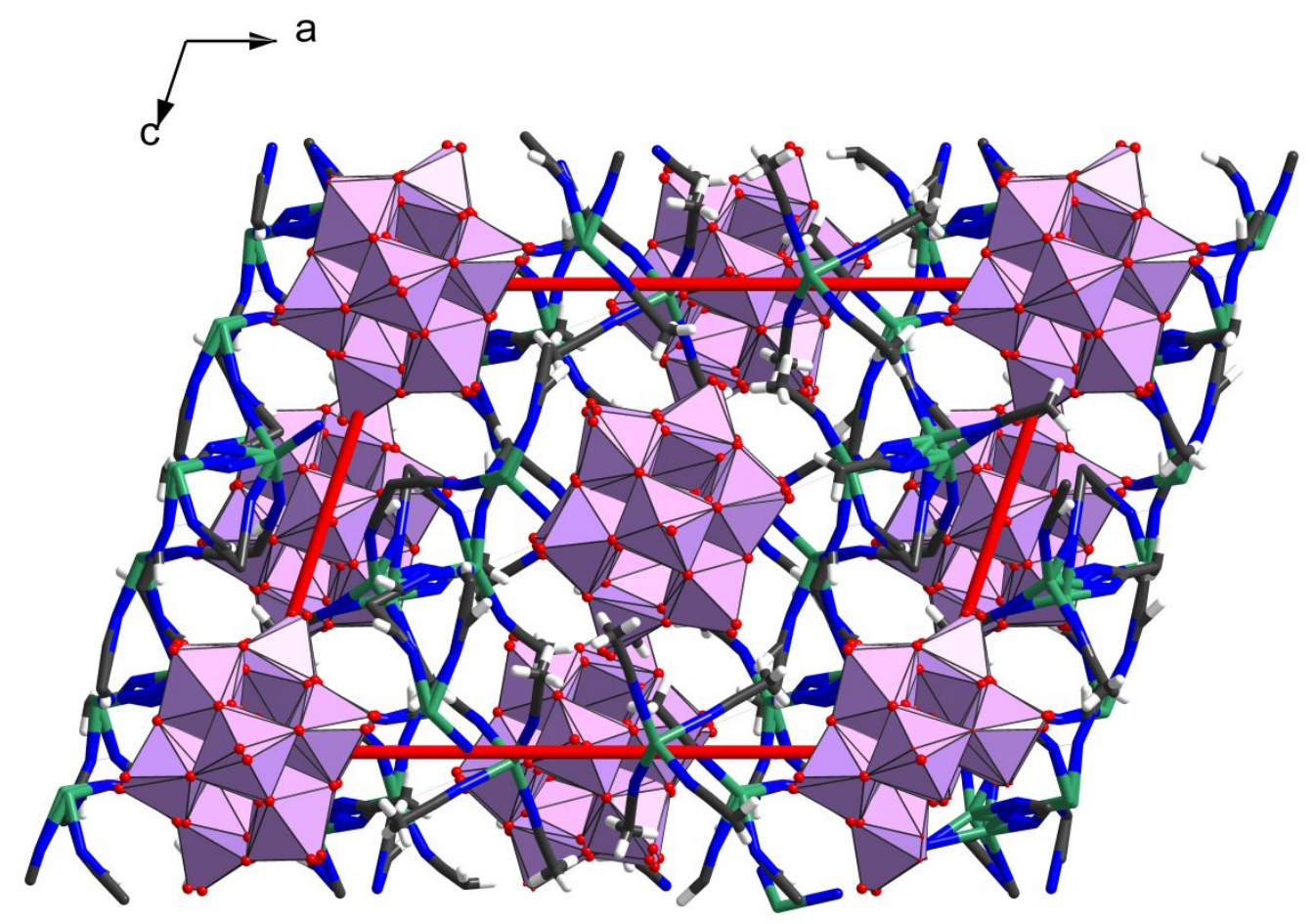

Figure S7. Crystal packing of 2 a along [010]. 


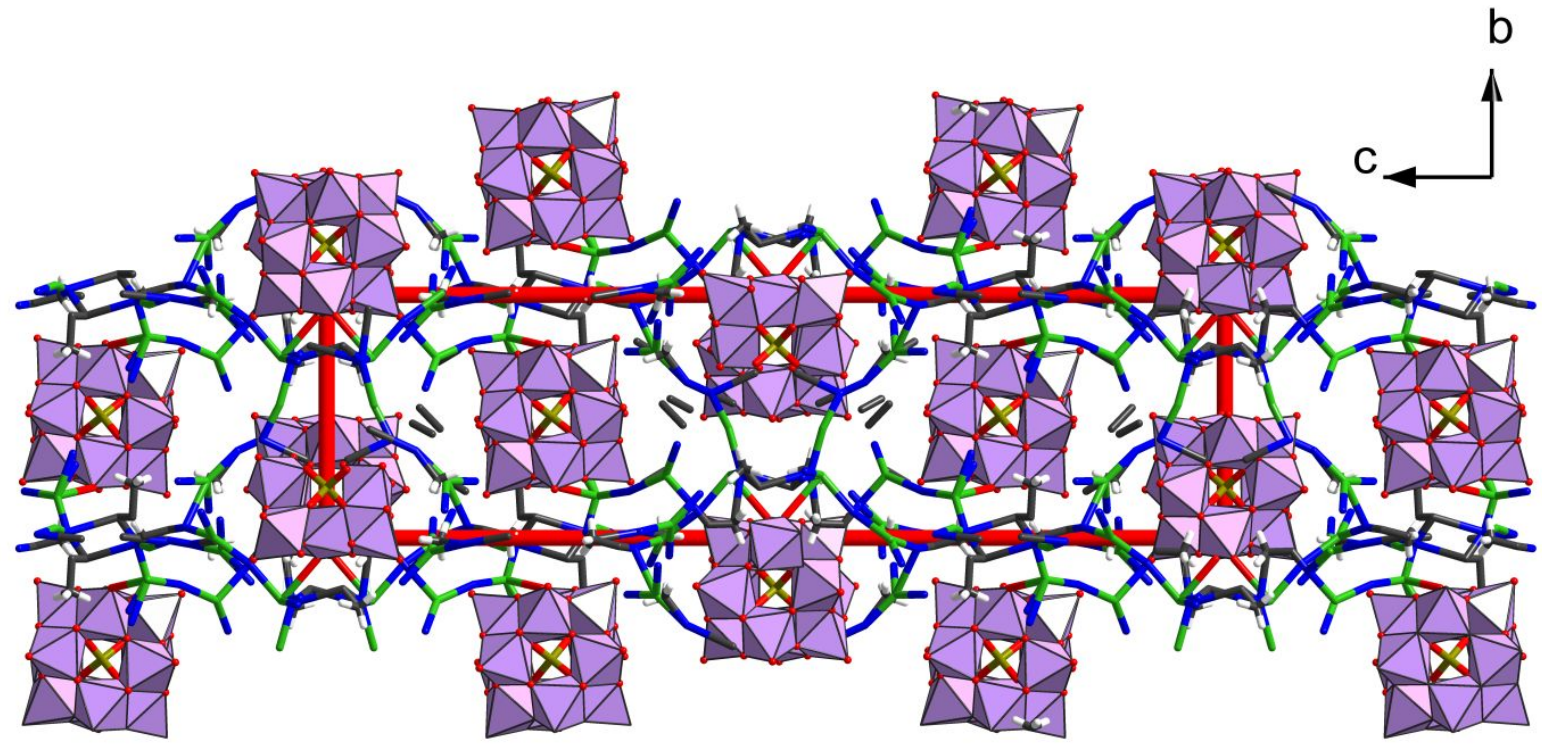

Figure S8. Crystal packing of 3a along [100]. 


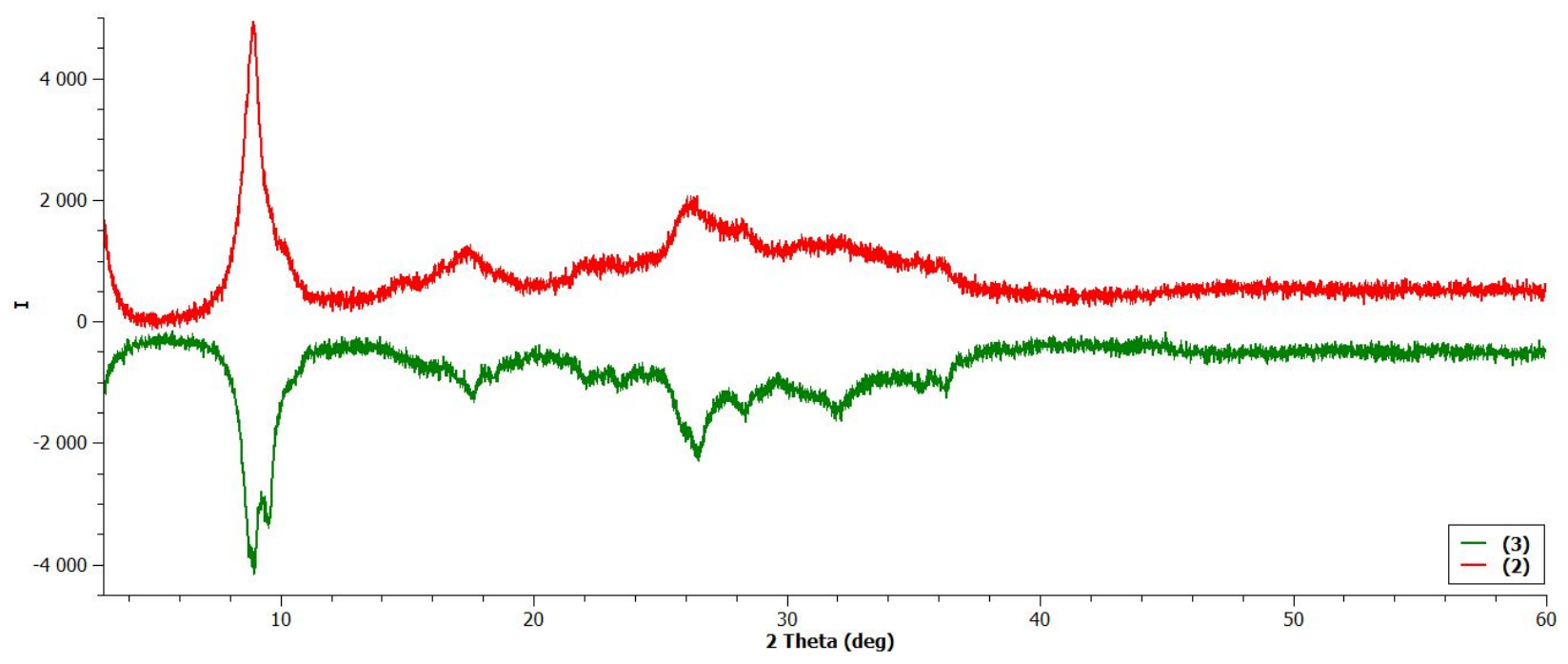

Figure S9. Comparison of complexes 2 and 3 XRPD patterns. Loosing of the crystallinity due to the $\mathrm{CH}_{3} \mathrm{CN}$ loss in both cases leads to widening of the diffraction peaks. 


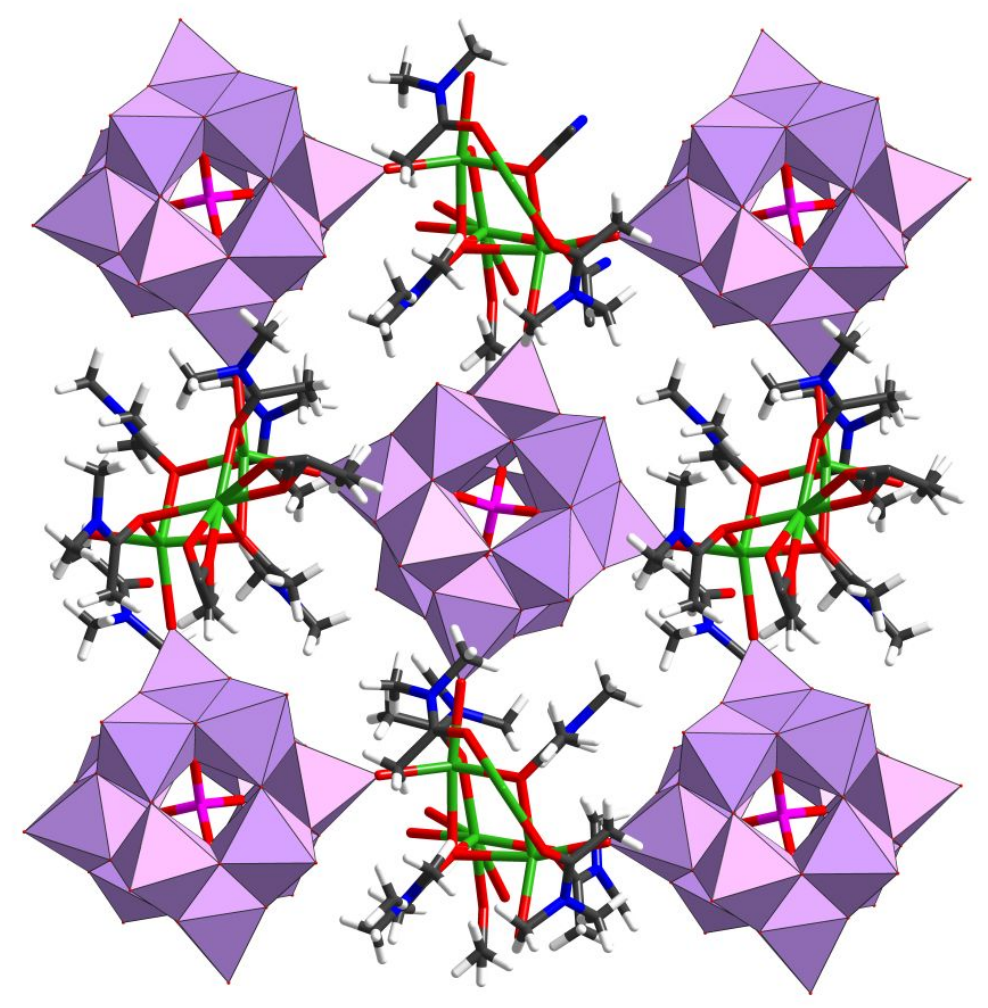

Figure S10. The topology of POM and $\left\{\mathrm{Ag}_{3}(\mathrm{DMA})_{6}\right\}^{3+}$ units connection in the crystal structure of 4 .

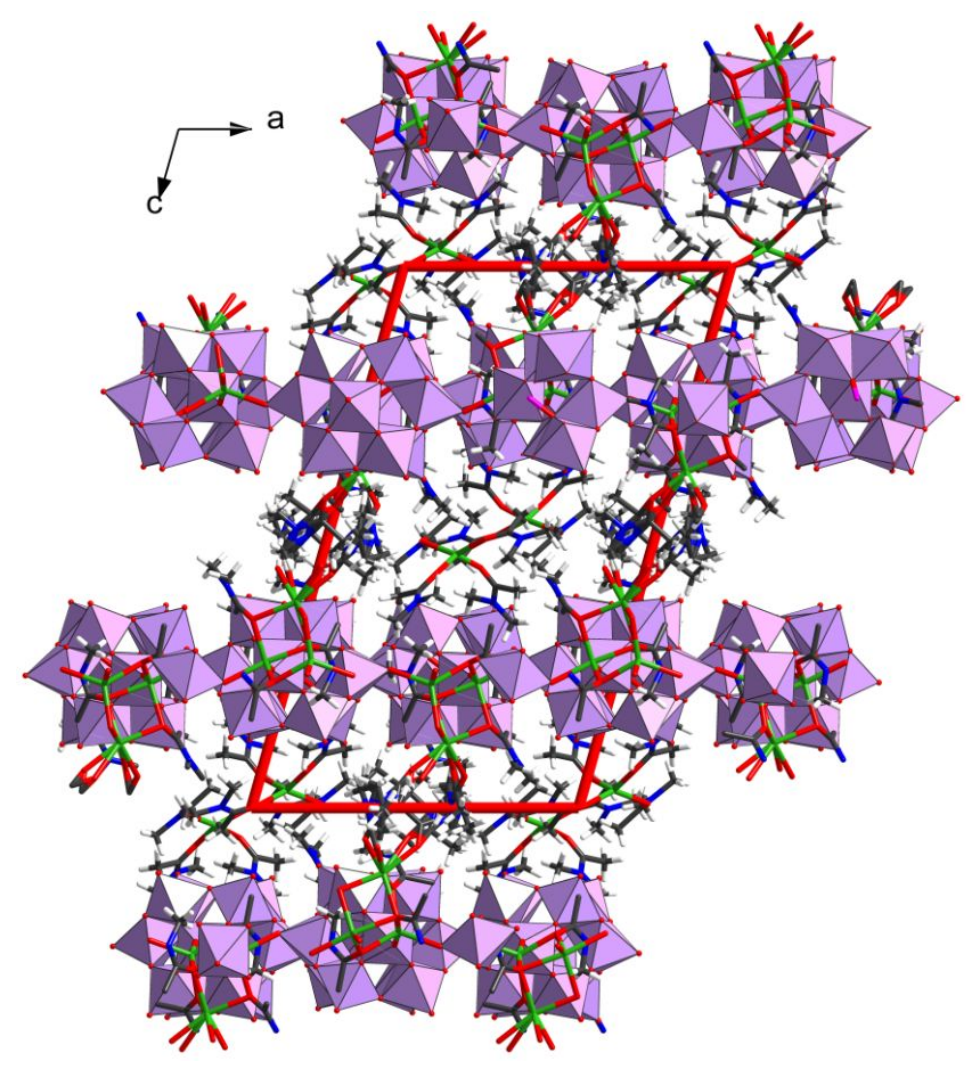

Figure S11. The layer topology of crystal packing of 4. 


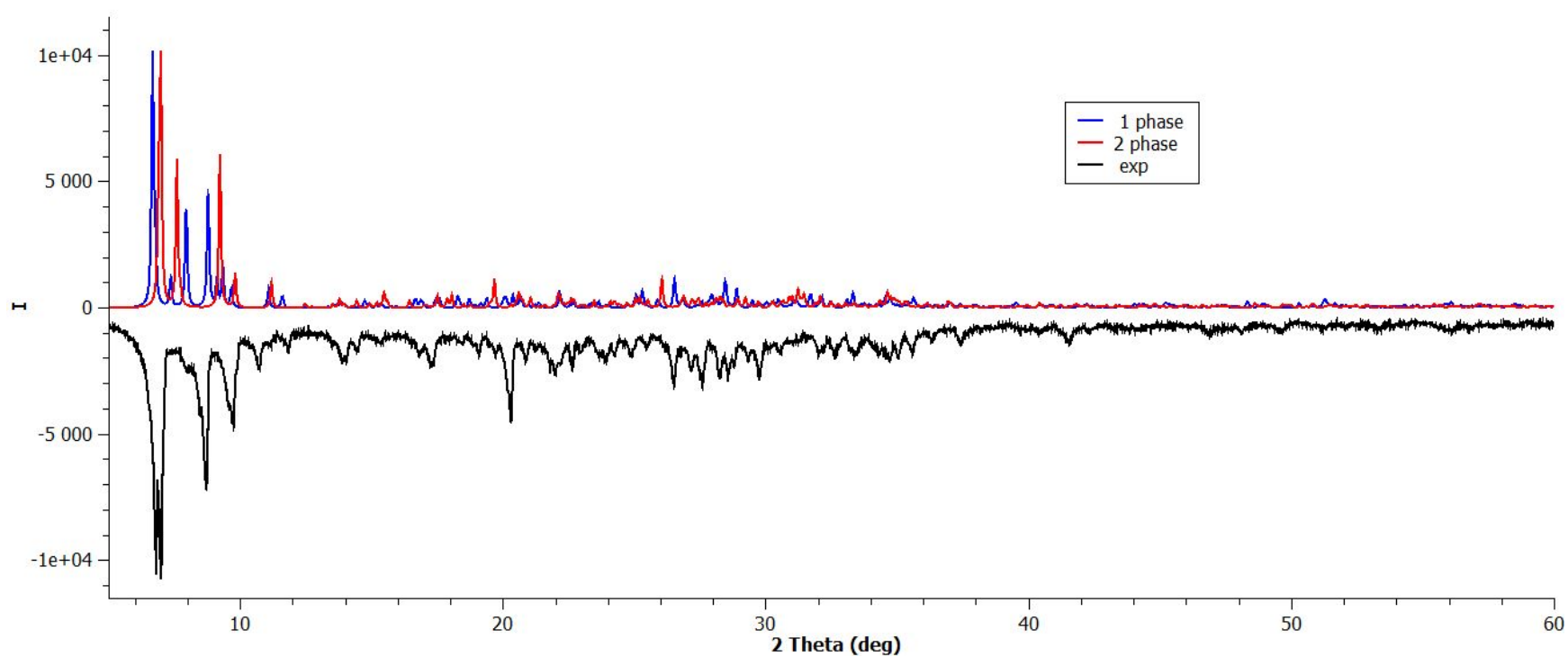

Figure S12. Comparison of experimental and calculated powder patterns for $5 \mathrm{a}$ and 5 b. 


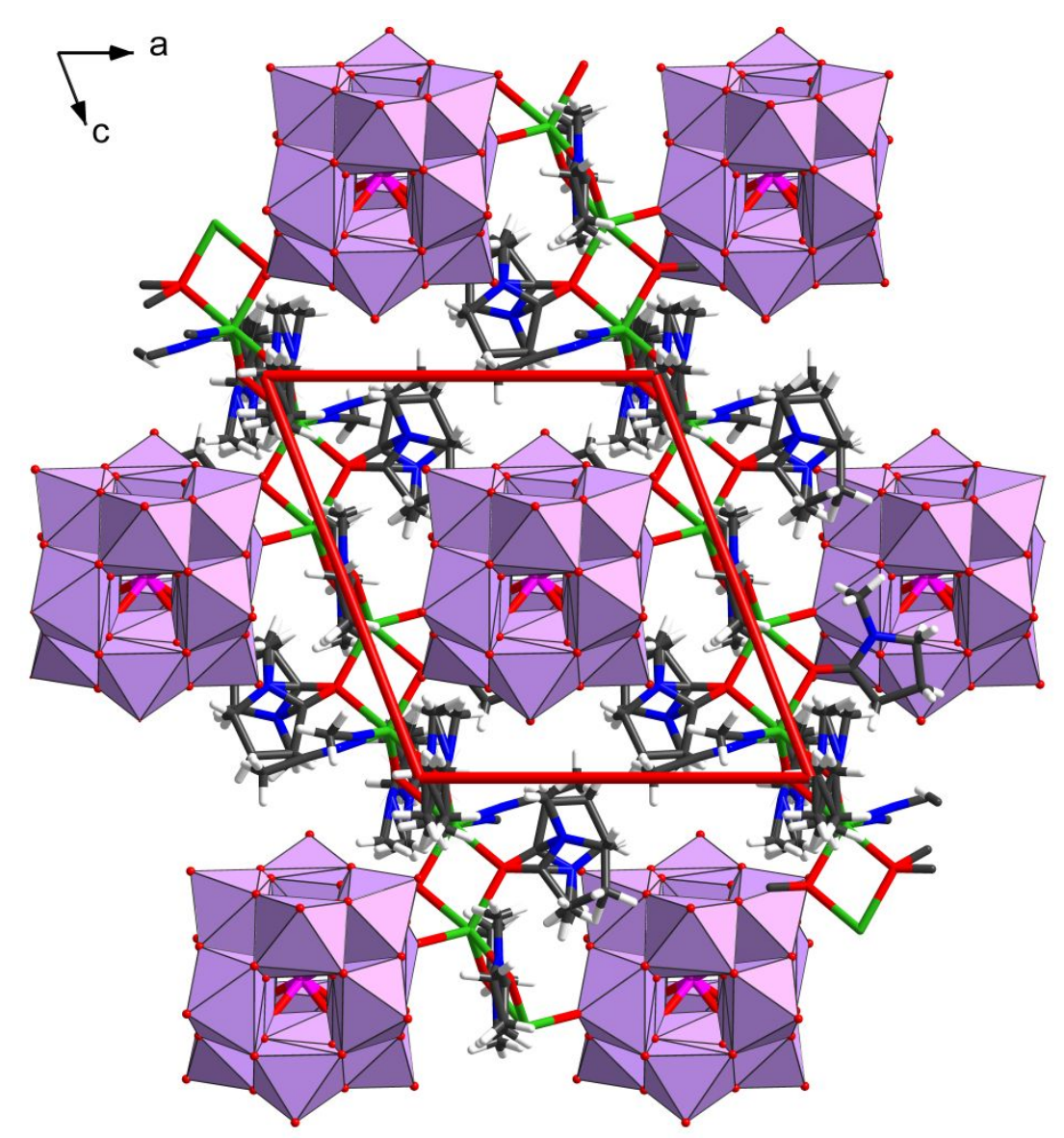

Figure S13. Packing of 5a along [010] direction.

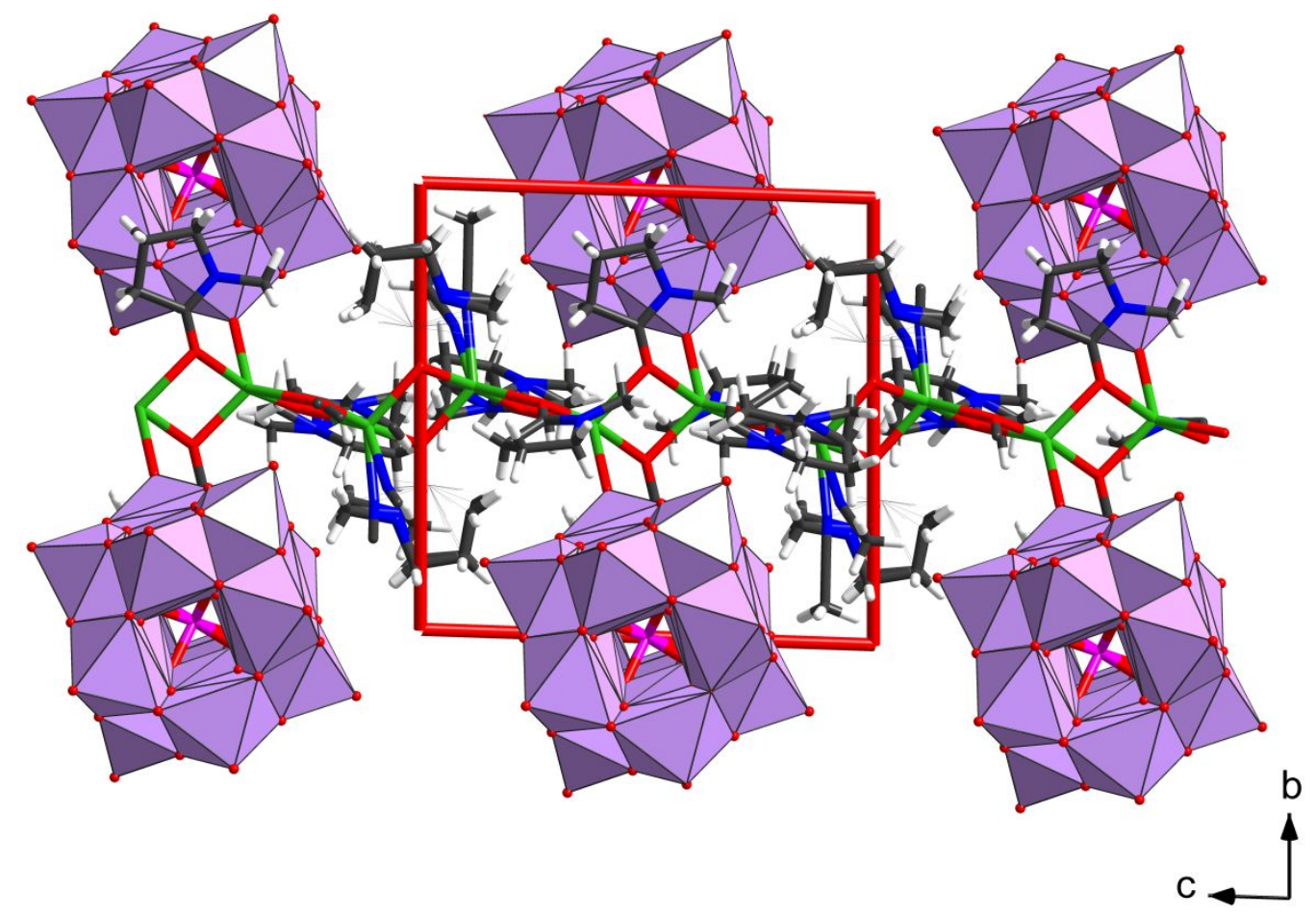

Figure S14. Packing of 5a along [100] direction. 


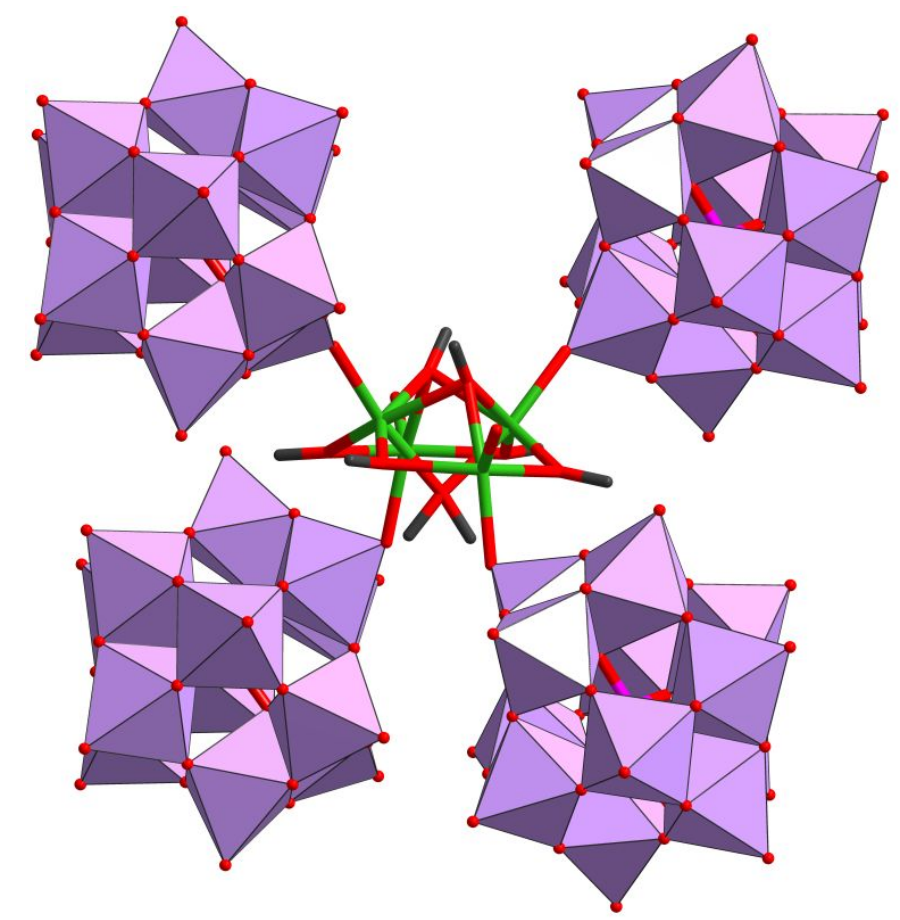

Figure S15. The tetrahedral orientation of POM anions around butterfly $\left\{\operatorname{Ag}_{4}(\mathrm{DEF})_{9}\right\}$ unit.

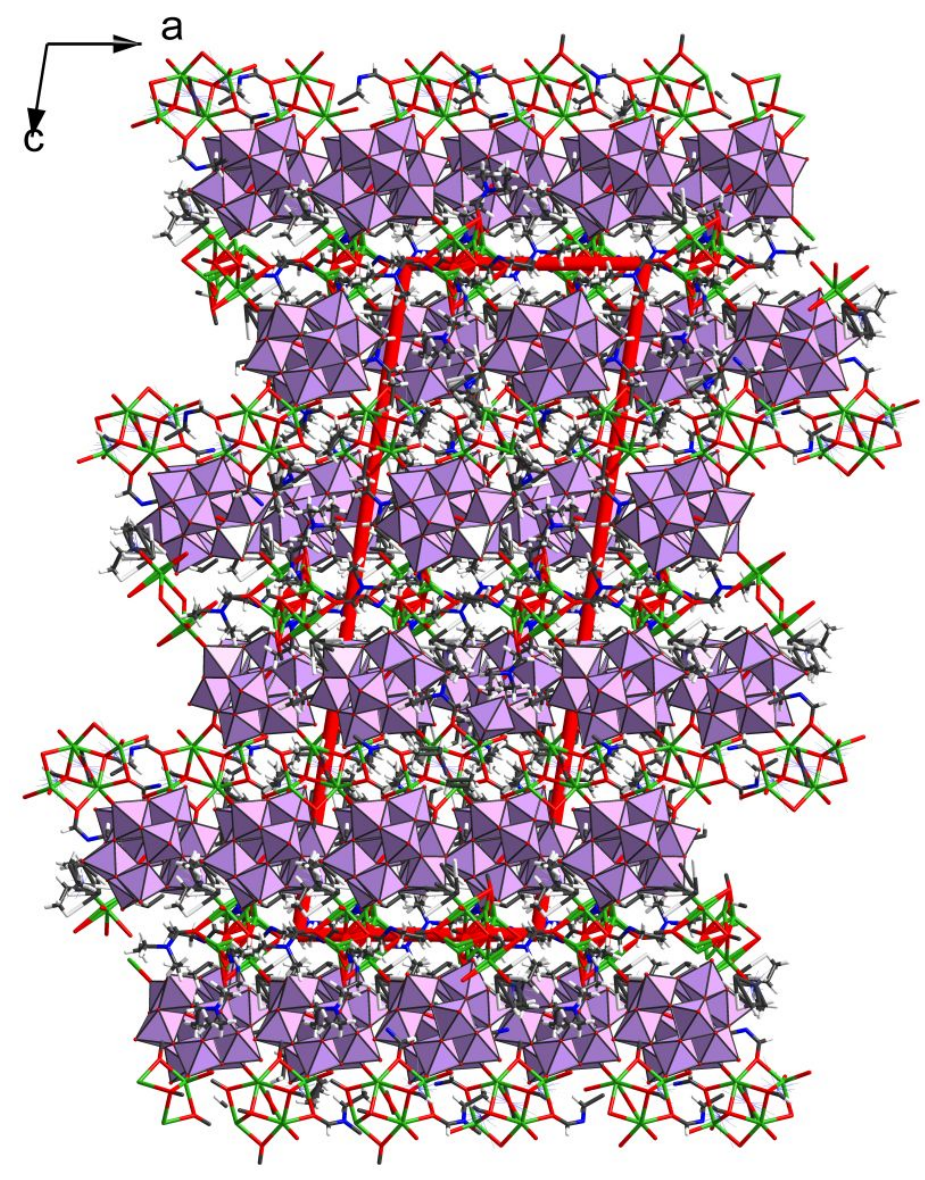

Figure S16. The crystal packing of 6. 


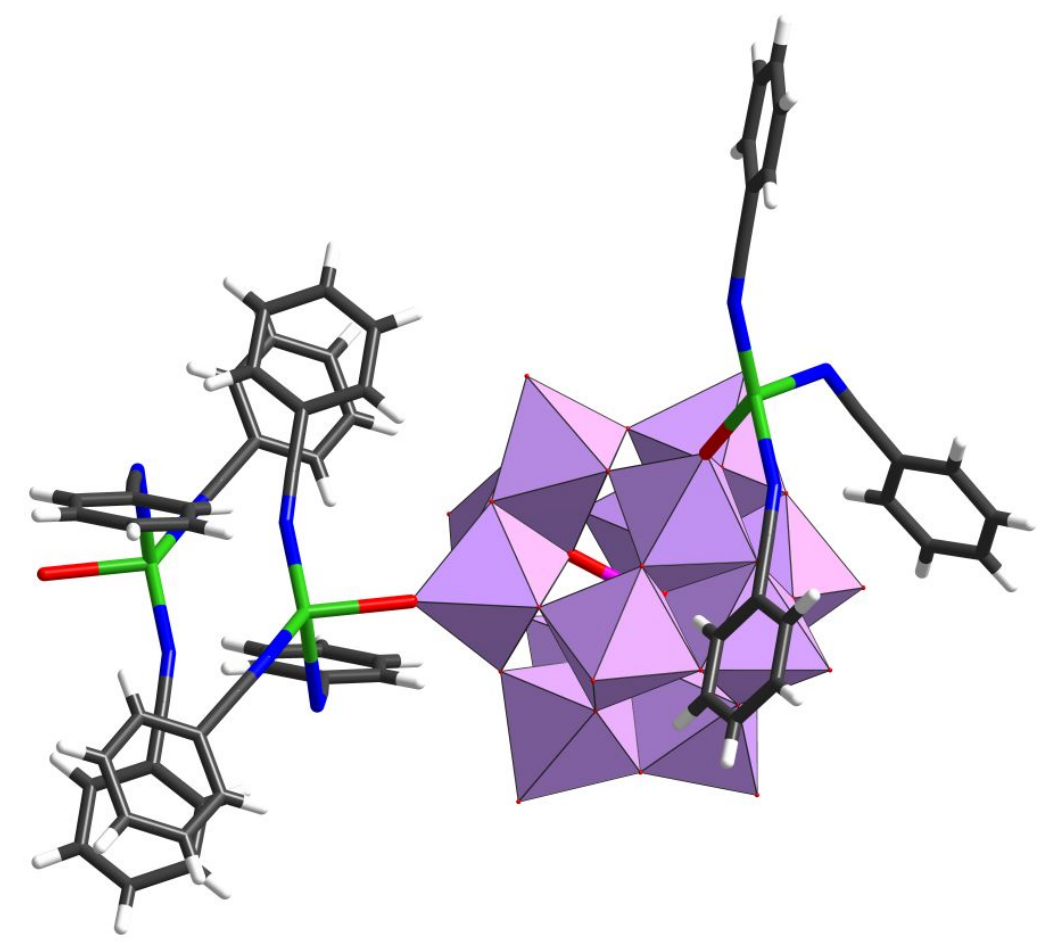

Figure S17. $\pi-\pi$ interactions between $\left[\left\{\mathrm{Ag}(\mathrm{PhCN})_{3}\right\}_{2} \mathrm{PW}_{11} \mathrm{NbO}_{40}\right]^{2-}$ in the crystal structure of 7 .

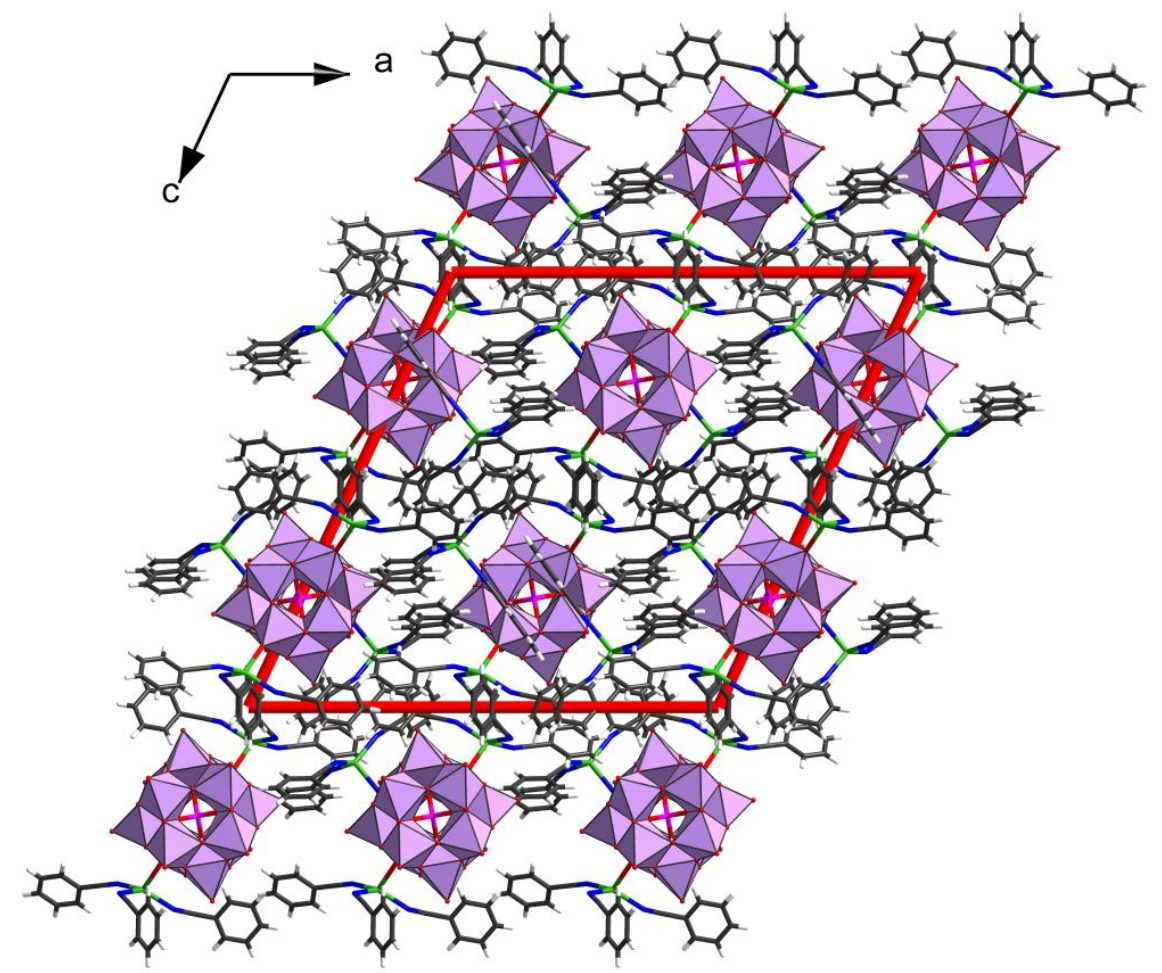

Figure S18. Crystal packing of 7. 


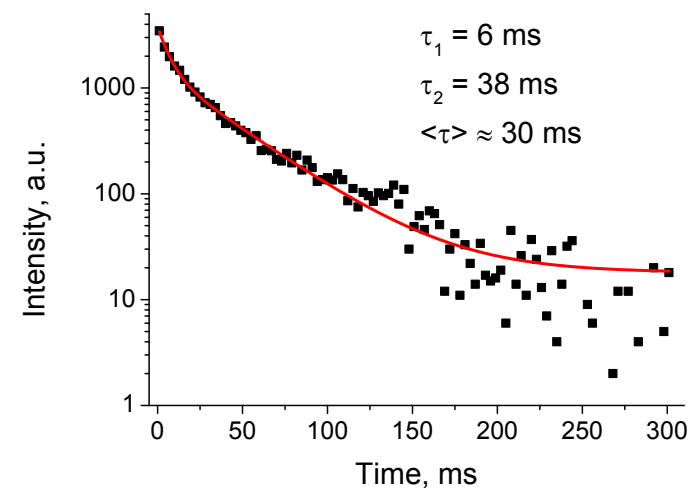

Figure S19. Kinetic of luminescence decay for powder $5 \mathrm{a} / 5 \mathrm{~b}$ at $77 \mathrm{~K}\left(\lambda_{\mathrm{Ex}}=390 \mathrm{~nm}\right.$, $\left.\lambda_{\mathrm{Em}}=535 \mathrm{~nm}\right)$.

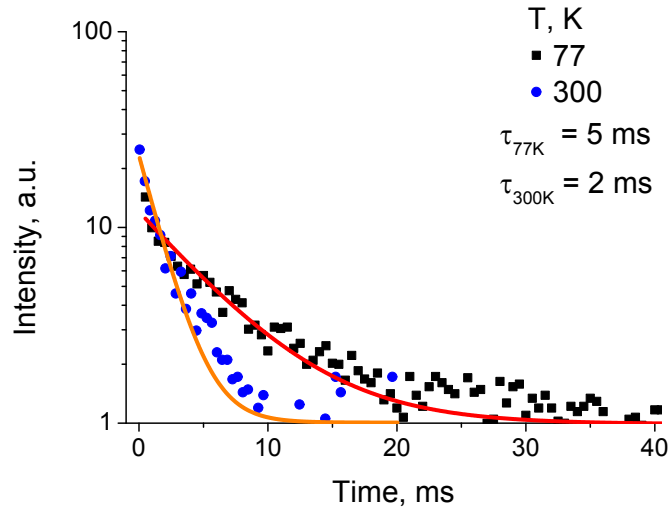

Figure S20. Kinetics of luminescence decay for powder 7 at $77 \mathrm{~K}$ and $300 \mathrm{~K}\left(\lambda_{\mathrm{Ex}}=\right.$ $370 \mathrm{~nm}, \lambda_{\mathrm{Em}}=540 \mathrm{~nm}$ ). 


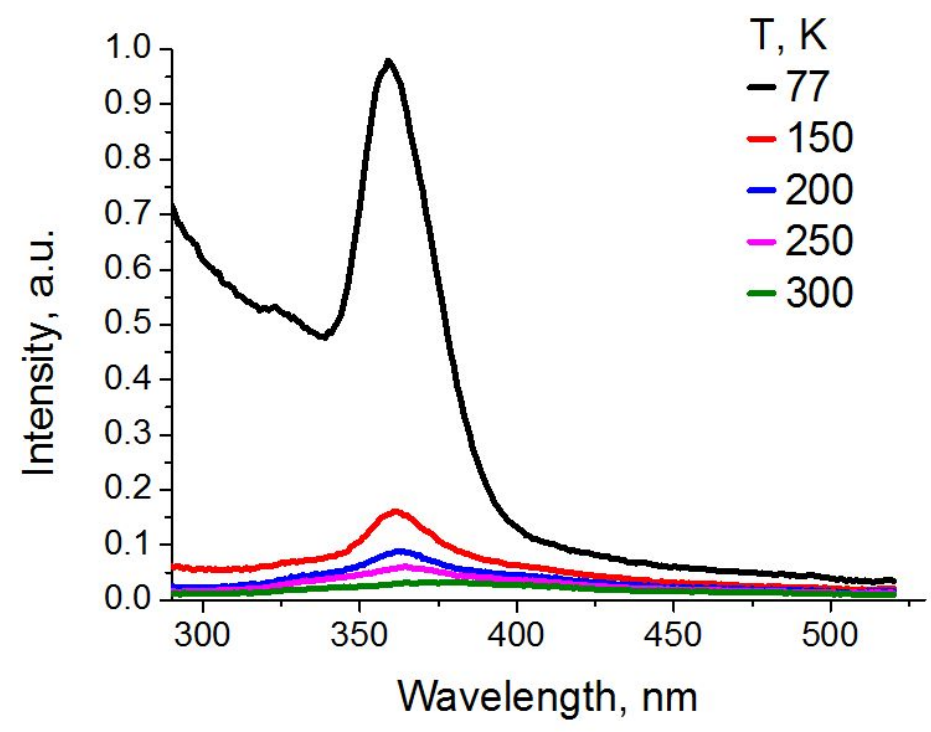

Figure S21. Temperature dependence of PLE $\left(\lambda_{E m}=540 \mathrm{~nm}\right)$ spectra of powder 7 . 